\title{
Constrained bounds on measures of entanglement
}

\author{
Animesh Datta.' Steven T. Flammia, Anil Shaji, and Carlton M. Caves \\ Department of Physics and Astronomy, University of New Mexico, \\ Albuquerque, New Mexico 87131-1156, USA.
}

(Dated: May 31, 2007)

\begin{abstract}
Entanglement measures constructed from two positive, but not completely positive maps on density operators are used as constraints in placing bounds on the entanglement of formation, the tangle, and the concurrence of $4 \times N$ mixed states. The maps are the partial transpose map and the $\Phi$-map introduced by Breuer [H.-P. Breuer, Phys. Rev. Lett. 97, 080501 (2006)]. The norm-based entanglement measures constructed from these two maps, called negativity and $\Phi$-negativity, respectively, lead to two sets of bounds on the entanglement of formation, the tangle, and the concurrence. We compare these bounds and identify the sets of $4 \times N$ density operators for which the bounds from one constraint are better than the bounds from the other. In the process, we present a new derivation of the already known bound on the concurrence based on the negativity. We compute new bounds on the three measures of entanglement using both the constraints simultaneously. We demonstrate how such doubly constrained bounds can be constructed. We discuss extensions of our results to bipartite states of higher dimensions and with more than two constraints.

PACS numbers: 03.67.Mn, 03.65.-w

Keywords: Entanglement Detection, Entanglement of Formation, Concurrence, Tangle, Entanglement Monotone, Negativity, Convex Roof
\end{abstract}

\section{INTRODUCTION}

Characterizing quantum entanglement [1, [2] is an important open problem in quantum information theory [3]. The nonclassical correlations associated with entanglement have been of immense interest since the very inception of quantum mechanics [4], 5. Quantum information science has identified entanglement as a potential resource. The ability of quantum computers to solve classically hard problems efficiently, the increased security of quantum cryptographic protocols, the enhanced capacity of quantum channels - all these are attributed to entanglement [3]. The presence of entanglement has been related to quantum phase transitions and the behavior of condensed systems [6], [7], 8]. Entanglement has also allowed the understanding of techniques such as density-matrix-renormalization group in a new light [9]. A significant part of recent research in theoretical quantum information science has centered around understanding and characterizing entanglement. In spite of this, entanglement remains a poorly understood feature of quantum systems.

Although many tests have been devised which attempt to decide whether a general quantum state is separable or not, this problem is known to be NP-Hard [10]. Quantifying entanglement

*Electronic address: animesh@unm.edu 
involves devising functions acting on quantum states that, in some reasonable way, order entangled states according to the degree of nonclassical correlation possessed by them. Measures of entanglement can be broadly divided into two classes depending on whether an efficient way of computing them for arbitrary states exists or not. Tests for separability can also be classified in a similar fashion [2]. Computationally operational measures of entanglement are easy to calculate for any state, while there is no known procedure for efficiently calculating computationally nonoperational measures for an arbitrary state. From here on we abbreviate the descriptions computationally operational and computationally nonoperational to simply operational and nonoperational, respectively. Several physically significant measures of entanglement are of the nonoperational variety. This makes it important to place bounds on the values of such measures. In this paper, we investigate the problem of placing lower bounds on nonoperational measures of entanglement for a quantum state assuming that we know the values of one or more operational measures for that state.

The outline of this paper is as follows. In Sec. II we start with examples of both operational and nonoperational measures of entanglement. We then discuss the general scheme of placing bounds on nonoperational measures using operational ones as constraints. In Sec. III we start with the separability criterion due to Breuer [11] and then show that a new, operational entanglement measure, called the $\Phi$-negativity, can be extracted from it. In Sec. [V] we use the $\Phi$-negativity to bound three nonoperational measures of entanglement for $4 \times N$ systems, namely, the entanglement of formation, the tangle, and the concurrence. We compare our results to the bounds based on another operational measure, the negativity. In the process, we present a different way of deriving the results in [12]. In Sec. $\mathrm{V}$ we obtain bounds on the three nonoperational measures using both the negativity and $\Phi$-negativity simultaneously as constraints. We also discuss how our new bounds relate to previously known bounds in this section. Our conclusions and future prospects are summarized in Sec. VI.

\section{GENERAL CONSIDERATIONS}

\section{A. Operational and nonoperational measures of entanglement}

A commonly used measure of entanglement for a pure-state $|\Psi\rangle$ of two systems $A$ and $B$ is the entropy of the reduced density operator $\rho_{A}$ (or $\rho_{B}$ ),

$$
S\left(\rho_{A}\right)=-\operatorname{Tr}\left(\rho_{A} \log \rho_{A}\right)=S\left(\rho_{B}\right)=-\operatorname{Tr}\left(\rho_{B} \log \rho_{B}\right)
$$

We write this entropy either as a function $h(\Psi)$ of the state $|\Psi\rangle$ or as a function $H(\boldsymbol{\mu})$ of the vector of Schmidt coefficients of $|\Psi\rangle$. It is a physically motivated quantity, in that it gives the rate at which copies of a pure state can be converted, by using only local operations and classical communication (LOCC), into copies of maximally entangled states and vice versa [13]. This measure can be elevated so that it applies to bipartite mixed states also by taking the so-called convex-roof extension of 
Eq. (2.1). This extended quantity is the entanglement of formation (EOF), and it is defined as

$$
\left.h(\rho) \equiv \min _{\left.\left\{p_{j}, \Psi_{j}\right\rangle\right\}}\left\{\sum_{j} p_{j} h\left(\Psi_{j}\right)\left|\rho=\sum_{j} p_{j}\right| \Psi_{j}\right\rangle\left\langle\Psi_{j}\right|\right\} .
$$

The EOF provides an upper bound on the rate at which maximally entangled states can be distilled from $\rho$ and a lower bound on the rate at which maximally entangled states must be supplied to create copies of $\rho$ [14]. Exact expressions for the EOF of several classes of states are known. One of the earliest, and simplest, was for an arbitrary state of two qubits [15]. The EOF in that case, was presented in terms of the concurrence, a subsidiary quantity. The concurrence itself has since been identified as an entanglement monotone and extended to higher-dimensional systems [16], [17].

The EOF and the concurrence are examples of a more general framework of defining entanglement measures. Suppose we have an entanglement measure $g$ defined only on pure states $|\Psi\rangle$, which is a concave function $G$ of Schmidt coefficients $\boldsymbol{\mu}$ of the marginal density operator of $|\Psi\rangle$. That is, suppose $g$ has the form $g(\Psi)=G(\boldsymbol{\mu})$ on pure states. This can be extended to a measure on mixed states via the convex-roof extension,

$$
\left.g(\rho)=\min _{\left\{p_{j},\left|\Psi_{j}\right\rangle\right\}}\left\{\sum_{j} p_{j} g\left(\Psi_{j}\right)\left|\rho=\sum_{j} p_{j}\right| \Psi_{j}\right\rangle\left\langle\Psi_{j}\right|\right\} .
$$

It has been proven [18] that any $g(\rho)$ constructed in this way is, on average, nonincreasing under LOCCs. An entanglement measure with this property is known as an entanglement monotone. Besides the EOF and concurrence, other examples of entanglement monotones include the tangle, relative entropy, entanglement of distillation, etc. Each has its use in particular physical contexts. All the entanglement measures just mentioned have one feature in common: they are nonoperational. The bottleneck in evaluating most of these measures for mixed states is the minimization over all pure-state decompositions. As a consequence, placing lower bounds on these measures of entanglement for arbitrary states becomes important.

An alternate approach to detecting and quantifying entanglement is based on the application of positive (but not completely positive) maps on density operators [19], 20], [21, [22], 223, [24], [25], [26], [27], [28]. In particular, a quantum state is separable if and only if it remains positive semidefinite under the action of any positive map. Given a positive map, we can construct an entanglement measure based on the spectrum of the density operators under the action of the map [29], [30]. Such measures are typically much easier to calculate for general quantum states than the ones discussed earlier because they do not involve the convex-roof construction. Measures of entanglement based on positive maps are therefore operational in nature. The negativity [31, 30] is an example of an entanglement measure of this sort, derived from the transpose map [32], 24].

We can use the operational measures of entanglement as constraints to obtain bounds on nonoperational, convex-roof-extended ones. The complexity of the minimization in Eq. (2.3) is reduced by solving it over a constrained set, instead of over all pure-state decompositions. This was done in [33], 12] for the EOF and the concurrence by minimizing over states with a given value of negativity. We turn now to describing the general procedure for constructing bounds based on the use of one or more operational entanglement measures as constraints. 


\section{B. Multiply-constrained bounds on nonoperational measures of entanglement}

Let $f_{1}, \cdots, f_{K}$ be $K$ operational measures used to characterize the entanglement in a bipartite system. Assume that they have values $\mathbf{n} \equiv\left(n_{1}, \ldots, n_{K}\right)$ for a state $\rho$. Their action on pure states can be expressed as functions of the Schmidt coefficients, i.e.,

$$
f_{i}(\Psi)=F_{i}(\boldsymbol{\mu}), \quad i=1, \cdots, K
$$

We are interested in a lower bound on the value of an independent, nonoperational measure $g$, which is a monotone defined on mixed states via the convex-roof construction. Let us assume that for the state $\rho$, the optimal pure-state decomposition with respect to $g$ is $\rho=\sum_{j} p_{j}\left|\Psi^{j}\right\rangle\left\langle\Psi^{j}\right|$. Then

$$
g(\rho)=\sum_{j} p_{j} g\left(\Psi^{j}\right)=\sum_{j} p_{j} G\left(\boldsymbol{\mu}^{j}\right) .
$$

Now define the function

$$
\widetilde{G}\left(m_{1}, \ldots, m_{K}\right) \equiv \widetilde{G}(\mathbf{m})=\min _{\boldsymbol{\mu}}\left\{G(\boldsymbol{\mu}) \mid F_{1}(\boldsymbol{\mu})=m_{1}, \ldots, F_{K}(\boldsymbol{\mu})=m_{K}\right\} .
$$

Let $\mathcal{G}(\mathbf{m})=\operatorname{co}[\widetilde{G}(\mathbf{m})]$ be the convex hull of $\widetilde{G}(\mathbf{m})$, i.e., the largest convex function of $K$ variables $\left(m_{1}, \ldots, m_{K}\right)$ that is bounded from above by $\widetilde{G}(\mathbf{m})$. Using Eq. $\sqrt{2.6}$ and the convexity of $\mathcal{G}$, we can write

$$
g(\rho) \geq \sum_{j} p_{j} \mathcal{G}\left(\mathbf{n}^{j}\right) \geq \mathcal{G}\left(\sum_{j} p_{j} \mathbf{n}^{j}\right) .
$$

If $\mathcal{G}$ is a monotonically nondecreasing function of all its arguments and if the operational measures $F_{i}$ are convex functions so that $\sum_{j} p_{j} n_{i}^{j} \geq n_{i}$, we obtain

$$
g(\rho) \geq \mathcal{G}(\mathbf{n})
$$

If the conditions for the validity of the inequality (2.8) are met, then we obtain a lower bound on $g(\rho)$ by knowing the operational measures $\mathbf{n}$ for $\rho$.

Regrettably, the first assumption leading to inequality $(2.8)$ is not always valid: the function $\mathcal{G}(\mathbf{n})$ is not guaranteed to be monotonic. If it is not, then we have to impose monotonicity by introducing a new monotonically nondecreasing function $\widetilde{G}_{\uparrow}(\mathbf{n})$, constructed from $\widetilde{G}(\mathbf{n})$. In the examples we consider in Sec. $\mathrm{V}, \widetilde{G}(\mathbf{n})$ turns out to be monotonic, so we do not have to construct the new function $\widetilde{G}_{\uparrow}(\mathbf{n})$. For the sake of completeness, the general construction of $\widetilde{G}_{\uparrow}(\mathbf{n})$ is presented in Appendix A

We can now redefine $\mathcal{G}(\mathbf{n})$ as the convex hull of $\widetilde{G}_{\uparrow}(\mathbf{n})$, rather than simply the convex hull of $\widetilde{G}(\mathbf{n})$. It is not immediately obvious that the convex hull of a monotonically nondecreasing function is also monotonically nondecreasing. The proof that this is so is given in Appendix B.

The only requirement on the operational entanglement measures $F_{i}$ for using them as constraints is that they are convex functions on the set of states. Furthermore, even if we do not have the functions $F_{i}$ themselves, but have instead functions $\hat{F}_{i}$ that bound $F_{i}$ from above for pure 
states, then the functions $\hat{F}_{i}$ can be used as constraints in the definition 2.6 of $\widetilde{G}$, in place of the functions $F_{i}$. The arguments leading to inequality $(2.8)$ go through exactly as before, i.e., $g(\rho) \geq \sum_{j} p_{j} \mathcal{G}\left(\hat{\mathbf{n}}^{j}\right) \geq \mathcal{G}\left(\sum_{j} p_{j} \hat{\mathbf{n}}^{j}\right) \geq \mathcal{G}\left(\sum_{j} p_{j} \mathbf{n}^{j}\right) \geq \mathcal{G}(\mathbf{n})$, the only difference being that there is an additional step, the second-to-last one, where we use $\hat{F}_{i}(\boldsymbol{\mu})=\hat{n}_{i} \geq n_{i}=F_{i}$ to conclude that $\sum_{j} p_{j} \hat{n}_{i}^{j} \geq \sum_{j} p_{j} n_{j}$. The danger in using upper bounds instead of the actual values of the functions $F_{i}$ is that the final bound on $g(\rho)$ might turn out to be less useful or even meaningless. In the example we consider in Sec. V] however, one of the constraints we use is an upper bound on an operational entanglement measure, rather than the measure itself, yet the bound we get turns out to be stronger than previous bounds.

Since our bound is intended for arbitrary states, there is one more subtlety to address, and that is the domain of the functions $G, \widetilde{G}$, and $\mathcal{G}$. The operational measures $\mathbf{n}$ map the state $\rho$ to a point in a $K$-dimensional hypercube in the space of the $K$ independent constraints $n_{k}$. Pure states correspond to a simply connected subset in this hypercube, which we call the pure-state region. The pure-state region is the domain of the functions $G$ and $\widetilde{G}$. This domain is not always convex, and so $\mathcal{G}(\mathbf{n})$ is defined on the convex hull of the pure-state region, which is generally bigger than the pure-state region, though only a subset of the full hypercube available to a general state.

Finally, we have to extend $\mathcal{G}(\mathbf{n})$ to the entire hypercube of states. Note that for inequalities (2.7) and (2.8) to hold, $\mathcal{G}(\mathbf{n})$ must be a monotonically nondecreasing function in the entire hypercube while it has to be convex only on the convex hull of the pure-state region. So, in extending $\mathcal{G}(\mathbf{n})$ outside the hull, we only have to take into account the monotonicity requirement (2.8). To construct such an extension of $\mathcal{G}(\mathbf{n})$, start from a point on the boundary of the hull and begin traversing out along decreasing directions parallel to the axes of the hypercube. Outside the hull, and till reaching the boundaries of the hypercube, the extension is defined as the constant function with value equal to that at the point on the boundary of the hull. To generate the complete extension, this simple procedure is repeated for every point on all the boundaries of the hull. This procedure is also demonstrated in detail in Sec. $\mathrm{V}$ for the example we consider.

In this paper, we carry out the general program just described with two particular constraints $(K=2)$. One of them is the negativity [30]. For the second, we develop a new entanglement measure, called $\Phi$-negativity, based on a recently presented separability criterion [11] (see [34] for another measure based on the same criterion). Like the negativity, it is easily computable for any $\rho$ and there are no convex-roof constructions involved in the computation. The $\Phi$-negativity, unlike the negativity, is not a simple function of the Schmidt coefficients for pure states. We find a simple function of the Schmidt coefficients that is an upper bound on the $\Phi$-negativity and, as described above, we use this function instead as the constraint to simplify our computations. We use both the (upper bound on) $\Phi$-negativity and the negativity simultaneously as constraints to place new bounds on the EOF, tangle, and concurrence of $4 \times N$ systems. Ours is the first instance of a doubly-constrained bound on entanglement measures for a family of states. It puts bounds that are tighter than those obtained in [33], [12. Multiply constrained bounds based on entanglement witnesses that can be applied to individual quantum states have been obtained using a different approach in [35], 36]. 
Although all of the results in this paper are obtained using the negativity and $\Phi$-negativity, a third constraint based on the realignment criterion [26], 27], 28] can be added to improve the bounds for certain classes of states. On pure states, the negativity and the realignment criterion lead to the same constraint. This means that in deriving both the singly and doubly constrained bounds we could have modified the negativity to take advantage of this, as was done in [33], [34]. Furthermore, the addition of the realignment criterion adds very little complexity to the procedure described below.

Before concluding this section, we review the notation used in this paper. We use lower case Latin letters, say $g$, to denote entanglement measures. The corresponding upper case character, $G$, denotes the same entanglement measure defined on pure states, expressed as a function of the Schmidt coefficients. The same letter with a tilde on top, $\widetilde{G}$, stands for the minimum of $G$ subject to constraints. Calligraphic letters like $\mathcal{G}$ denote the bound on $g$ obtained by taking the convex hull of $\widetilde{G}$. If we have to impose monotonicity on $\widetilde{G}$ as an intermediate step, we define a new function $\widetilde{G}_{\uparrow}$.

\section{III. $\Phi$-MAP}

Recently, a new separability criterion has been proposed based on a positive nondecomposable map [11. It is a combination of the Peres criterion and the reduction criterion [37], [38] for detecting entangled states. In this section we construct a new entanglement measure from this map and calculate it for pure states.

\section{A. Separability criterion}

Let us consider a finite-dimensional Hilbert space $\mathbb{C}^{D}$. It can be regarded as the space of a spin- $j$ particle with $D=2 j+1$. A natural basis for this space is the "angular-momentum basis" $|j, m\rangle$, where $m=-j,-j+1, \ldots, j-1, j$. The separability criterion to be presented involves the time-reversal operator $\vartheta$ whose action on an operator $\sigma$ acting on $\mathbb{C}^{D}$ is given as

$$
\vartheta \sigma=V \sigma^{T} V^{\dagger},
$$

where the superscript $T$ stands for transposition in the angular-momentum basis and $V$ is a unitary operator defined as

$$
\left\langle j, m|V| j, m^{\prime}\right\rangle=(-1)^{j-m} \delta_{m,-m^{\prime}}
$$

This map was initially introduced by Breuer to study the entanglement of $4 \times 4 \mathrm{SU}(2)$ invariant states; in that case, the $\vartheta$ map, together with the Peres criterion, was found to be a necessary and sufficient separability condition [39]. In even dimensions, an additional property holds: $V^{T}=-V$, i.e., $V$ is skew-symmetric in addition to being unitary.

The condition for positivity under the partial time-reversal map $(I \otimes \vartheta) \rho \geq 0$ is unitarily equivalent to the Peres PPT criterion $(I \otimes T) \rho \geq 0$. This means that partial time reversal can be used as an entanglement detection criterion. Breuer [11] defines a positive map

$$
\Phi(\rho)=\operatorname{Tr}(\rho) I-\rho-V \rho^{T} V^{\dagger},
$$


which conjoins the time reversal map with the so-called reduction criterion [37]. The map $\Phi$ then defines for any joint density operator $\rho_{A B}$ a necessary condition for separability as

$$
(I \otimes \Phi) \rho_{A B}=\operatorname{Tr}_{B}\left(\rho_{A B}\right) \otimes I_{B}-\rho_{A B}-\left(I_{A} \otimes V\right) \rho_{A B}^{T_{B}}\left(I_{A} \otimes V^{\dagger}\right) \geq 0 .
$$

Any state that violates the above condition must be entangled.

Consider the space $\mathcal{H}_{A} \otimes \mathcal{H}_{B}=\mathbb{C}^{D} \otimes \mathbb{C}^{D}$. It can be regarded, without loss of generality, as the Hilbert space of two spin- $j$ particles with $j=(D-1) / 2$. The total spin of the system, denoted by $J$ ranges over the values $J=0,1, \ldots, 2 j=D-1$. Let $P_{J}$ be the projector onto the $(2 J+1)$ dimensional spin- $J$ manifold. It can then be shown that $\Phi$ is a nondecomposable positive, but not completely positive map [11, [34] in all even dimensions $D$ greater than or equal to 4 . The proof of positivity cannot be extended to odd dimensions as it exploits the skew-symmetric nature of the unitary operator $V$. In addition, the hermitian operator

$$
W \equiv(I \otimes \Phi) P_{0}
$$

is an optimal entanglement witness [40], [11, in that the set of PPT states detected by $W$ is not contained in the set detected by any other single witness. There, of course, exist families of PPT states that $W$ fails to detect. The optimal nature of $W$ provides motivation for contructing a measure of entanglement based on the $\Phi$-map.

\section{B. Entanglement measures from maps}

Our endeavor here is to define a quantitative operational measure of entanglement based on the $\Phi$ map. We call this quantity the $\Phi$-negativity, denote it by $n_{\Phi}$, and define it for a general mixed state as

$$
n_{\Phi}(\rho)=\frac{D(D-1)}{4}\left[\frac{\|(I \otimes \Phi) \rho\|}{D-2}-1\right]
$$

where $D=\min \left(\operatorname{dim}\left(\mathcal{H}_{A}\right), \operatorname{dim}\left(\mathcal{H}_{B}\right)\right)$, and the trace norm of an operator is defined as $\|O\|=$ $\operatorname{Tr}\left(\sqrt{O O^{\dagger}}\right)$. For a separable state $\sigma,(I \otimes \Phi) \sigma$ has no negative eigenvalues, so $\|(I \otimes \Phi) \sigma\|=$ $\operatorname{Tr}[(I \otimes \Phi) \sigma]=(D-2) \operatorname{Tr}(\sigma)=D-2$. Hence the $\Phi$-negativity is zero on separable states. This calculation also shows that $\Phi$ is not trace preserving, and this motivates the factor of $D-2$ in the denominator of Eq. (3.6). The $\Phi$-negativity is a shifted and scaled version of the sum of the negative eigenvalues of a state under the action of the map (3.4). Since this sum can be expressed in terms of the trace norm of an operator, $\left\|(I \otimes \Phi) \rho_{A B}\right\|$, it is a convex function of $\rho$ as required in the general scheme described in Sec. III. By defining the $\Phi$-negativity in terms of a map, we make sure that it is an operational measure that involves no convex-roof extensions.

Similar measures of entanglement based on other positive, but not completely positive maps have previously been proposed and investigated [29]. The negativity [30], which is based on the Peres partial transpose criterion, is defined as

$$
n_{T}(\rho)=\frac{\left\|\rho^{T_{A}}\right\|-1}{2}
$$


where $\rho$ is a joint density operator, $T_{A}$ is the partial transposition with respect to system $A$. A positive value of $n_{T}$ indicates an entangled state. The Peres negativity, in addition to being a measure of entanglement, is also an entanglement monotone, since it is nonincreasing on average under LOCC operations [30]. The $\Phi$-negativity is not an entanglement monotone, but it is a convex function of $\rho$.

The $\Phi$-negativity is a new operational measure of entanglement for any quantum state. To use the $\Phi$-negativity as a constraint in bounding nonoperational measures we need expressions for $n_{\Phi}$ for pure states. We start from the Schmidt decomposition of any pure state,

$$
\left|\Psi_{A B}\right\rangle=\sum_{i=1}^{D} \sqrt{\mu_{i}}\left|a_{i}, b_{i}\right\rangle
$$

for $\left|\Psi_{A B}\right\rangle \in \mathbb{C}^{D} \otimes \mathbb{C}^{N}$ and $D \leq N$. The $\mu_{i}$ are the Schmidt coefficients, satisfying $\mu_{i} \geq 0 \forall i$ and $\sum_{i=1}^{D} \mu_{i}=1$.

Before we apply the map $(I \otimes \Phi)$ to this state we note that the matrix $V$ appearing in the definition of the $\Phi$-map has the form given in Eq. (3.2) only in the angular-momentum basis for system $B$, and the required transposition is also carried out in this basis. Relabeling the angularmomentum eigenvectors $\{|j, m\rangle\}$ as $\{|l\rangle\}$ with $l=1, \ldots, D=2 j+1$, we transform $\left|\Psi_{A B}\right\rangle$ to the angular-momentum basis for subsystem $B$ and obtain

$$
\rho_{A B}=\left|\Psi_{A B}\right\rangle\left\langle\Psi_{A B}\left|=\sum_{i, j, l, m=1}^{D} \sqrt{\mu_{i} \mu_{j}}\left\langle l \mid b_{i}\right\rangle\left\langle b_{j} \mid m\right\rangle\right| a_{i}, l\right\rangle\left\langle a_{j}, m\right|,
$$

and

$$
\begin{aligned}
(I \otimes \Phi) \rho_{A B}= & \sum_{i=1}^{D} \mu_{i}\left|a_{i}\right\rangle\left\langle a_{i}\left|\otimes \sum_{l=1}^{D}\right| l\right\rangle\langle l| \\
& -\sum_{i, j, l, m=1}^{D} \sqrt{\mu_{i} \mu_{j}}\left\langle l \mid b_{i}\right\rangle\left\langle b_{j} \mid m\right\rangle\left|a_{i}, l\right\rangle\left\langle a_{j}, m\right| \\
& -\sum_{i, j, l, m=1}^{D} \sqrt{\mu_{i} \mu_{j}}(-1)^{l+m}\left\langle b_{i} \mid l\right\rangle\left\langle m \mid b_{j}\right\rangle\left|a_{i}, D+1-m\right\rangle\left\langle a_{j}, D+1-l\right| .
\end{aligned}
$$

The trace norm $\left\|(I \otimes \Phi) \rho_{A B}\right\|$ and the entanglement measure $n_{\Phi}$ defined using the trace norm are rather complicated functions of the Schmidt coefficients $\mu_{i}$ and the matrix elements $\left\langle l \mid b_{i}\right\rangle$ of the unitary matrix that transforms between the Schmidt basis of subsystem $B$ the angular momentum basis used in Eq. (3.2). Computing the numerical value of $n_{\Phi}$ for any state is relatively easy, but the analytic expression for the entanglement measure is quite unwieldy.

All we really need to generate a constraint from the $\Phi$-map, which can be used to place lower bounds on nonoperational measures of entanglement, is an upper bound on $n_{\Phi}$. Such a bound is obtained by considering the special case in which the Schmidt basis of subsystem $B$ is the same as 
the basis of the angular-momentum eigenstates $\{|l\rangle\}$, i.e., $\left\langle l \mid b_{i}\right\rangle=\delta_{l i}$. We then have

$$
\begin{aligned}
(I \otimes \Phi) \rho_{A B}= & \sum_{i=1}^{D} \mu_{i}\left|a_{i}\right\rangle\left\langle a_{i}\left|\otimes \sum_{j=1}^{D}\right| b_{j}\right\rangle\left\langle b_{j}\right| \\
& -\sum_{i, j=1}^{D} \sqrt{\mu_{i} \mu_{j}}\left[\left|a_{i} b_{i}\right\rangle\left\langle a_{j} b_{j}\left|+(-1)^{i+j}\right| a_{i}\right\rangle\left\langle a_{j}|\otimes| b_{D-j+1}\right\rangle\left\langle b_{D-i+1}\right|\right] .
\end{aligned}
$$

For the first nontrivial case, $D=4$, which we will be using extensively, explicit diagonalization of the above operator is possible. As shown in Appendix C, it has six nonzero eigenvalues, one of which is negative. The trace norm can then be evaluated as the sum of the absolute values of the eigenvalues. Thus,

$$
\left\|(I \otimes \Phi) \rho_{A B}\right\|=\operatorname{Tr}\left(\sqrt{\left[(I \otimes \Phi) \rho_{A B}\right]^{2}}\right)=2\left[1+\sqrt{\left(\mu_{1}+\mu_{4}\right)\left(\mu_{2}+\mu_{3}\right)}\right],
$$

where we use the fact that the $\Phi$-map is hermiticity preserving. Therefore, for all $4 \times N$ pure states that have the Schmidt basis for subsystem $B$ the same as the angular-momentum basis,

$$
n_{\Phi}=3 \sqrt{\left(\mu_{1}+\mu_{4}\right)\left(\mu_{2}+\mu_{3}\right)} \equiv \hat{n}_{\Phi} .
$$

In Eqs. (3.12) and (3.13) and in all our subsequent discussion of the function $\hat{n}_{\Phi}$, the Schmidt coefficients are ordered from largest to smallest, i.e., $\mu_{1} \geq \mu_{2} \geq \mu_{3} \geq \mu_{4}$.

The function $\hat{n}_{\Phi}$ is a simple function of the Schmidt coefficients for any $4 \times N$ pure state and as shown numerically by the results displayed in Fig. 1, the true $\Phi$-negativity, $n_{\Phi}$, calculated with respect to a fixed angular-momentum basis, is always bounded from above by $\hat{n}_{\phi}$.

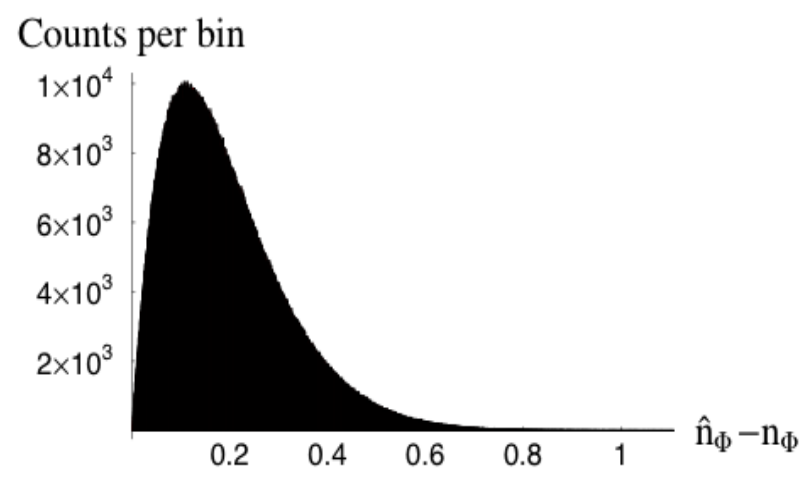

FIG. 1: A histogram of the difference $\hat{n}_{\Phi}-n_{\Phi}$ for five million $4 \times 4$ pure states picked randomly from the Haar measure. The bin size in the histogram is 0.001 . The difference is always found to be positive. These results carry over to $4 \times N$ states when $N>4$ ( $N$ even), since the difference only depends on the Schmidt coefficients. We have tried to prove that the difference is nonnegative without success and thus rely on this numerical demonstration instead.

In the rest of this paper we use $\hat{n}_{\Phi}$ instead of $n_{\Phi}$ as the constraint for bounding nonoperational measures because of its simple algebraic form. When we refer to constraints based on the $\Phi$ negativity we are referring to fixing the value of $\hat{n}_{\Phi}$. Expressions for $\hat{n}_{\Phi}$ for pure states in higher dimensions are discussed in Appendix C. 
In the next section, we will use $\hat{n}_{\Phi}$ to put lower bounds on the EOF, tangle, and concurrence for $4 \times N$ mixed states. We then compare our results to such bounds that have already been derived based on the Peres negativity $n_{T}$. For $D \times N$ pure states, the Peres negativity is given by [31, 30]

$$
n_{T}=\frac{\left(\sum_{i=1}^{D} \sqrt{\mu_{i}}\right)^{2}-1}{2} .
$$

\section{SINGLY CONSTRAINED BOUNDS}

\section{A. Entanglement of formation}

A lower bound $\mathcal{H}\left(\hat{n}_{\Phi}\right)$ on the EOF, constrained by pure states having a certain value for $\hat{n}_{\Phi}$, can be obtained using the steps described in Sec. II. All the subsequent results presented in this section and the next are for $4 \times N$ states $\rho$, with $N \geq 4$.

Firstly, we have to find

$$
\widetilde{H}\left(\hat{n}_{\Phi}\right)=\min _{\boldsymbol{\mu}}\left\{H(\boldsymbol{\mu}) \mid 3 \sqrt{\left(\mu_{1}+\mu_{4}\right)\left(\mu_{2}+\mu_{3}\right)}=\hat{n}_{\Phi}\right\},
$$

and then its convex hull,

$$
\mathcal{H}(\rho)=\operatorname{co}\left[\widetilde{H}\left(\hat{n}_{\Phi}\right)\right]
$$

provided $\widetilde{H}\left(\hat{n}_{\Phi}\right)$ is a monotonically increasing function of $\hat{n}_{\Phi}$. Defining $\mu_{1}+\mu_{4}=\alpha$ and $\mu_{2}+\mu_{3}=\beta$, we can write the normalization and $\hat{n}_{\Phi}$ constraints as

$$
\alpha+\beta=1 \quad \text { and } \quad \alpha \beta=\frac{\hat{n}_{\Phi}^{2}}{9},
$$

which give

$$
\alpha=\frac{1 \pm \sqrt{1-4 \hat{n}_{\Phi}^{2} / 9}}{2} \text { and } \beta=\frac{1 \mp \sqrt{1-4 \hat{n}_{\Phi}^{2} / 9}}{2}
$$

Minimizing

$$
H(\boldsymbol{\mu})=-\mu_{1} \log \mu_{1}-\mu_{4} \log \mu_{4}-\mu_{2} \log \mu_{2}-\mu_{3} \log \mu_{3}=H_{2}(\alpha)+\alpha H_{2}\left(\mu_{1} / \alpha\right)+\beta H_{2}\left(\mu_{2} / \beta\right)
$$

where $H_{2}(\cdot)$ is the binary entropy function, is trivial, because we simply make the last two terms zero by choosing $\mu_{1}=\alpha$ and $\mu_{2}=\beta$ [and choosing the upper sign in Eq. 4.4) so as to be consistent with the assumed ordering of the Schmidt coefficients]. Then the minimum entropy is

$$
\widetilde{H}\left(\hat{n}_{\Phi}\right)=H_{2}(\alpha)
$$

That $\widetilde{H}\left(\hat{n}_{\Phi}\right)$ is a convex, monotonically increasing function of $n_{\Phi}$ can be shown by considering its first and second derivatives. Its convex roof is the function itself, i.e.,

$$
\mathcal{H}\left(\hat{n}_{\Phi}\right)=\operatorname{co}\left[\widetilde{H}\left(\hat{n}_{\Phi}\right)\right]=\widetilde{H}\left(\hat{n}_{\Phi}\right)
$$



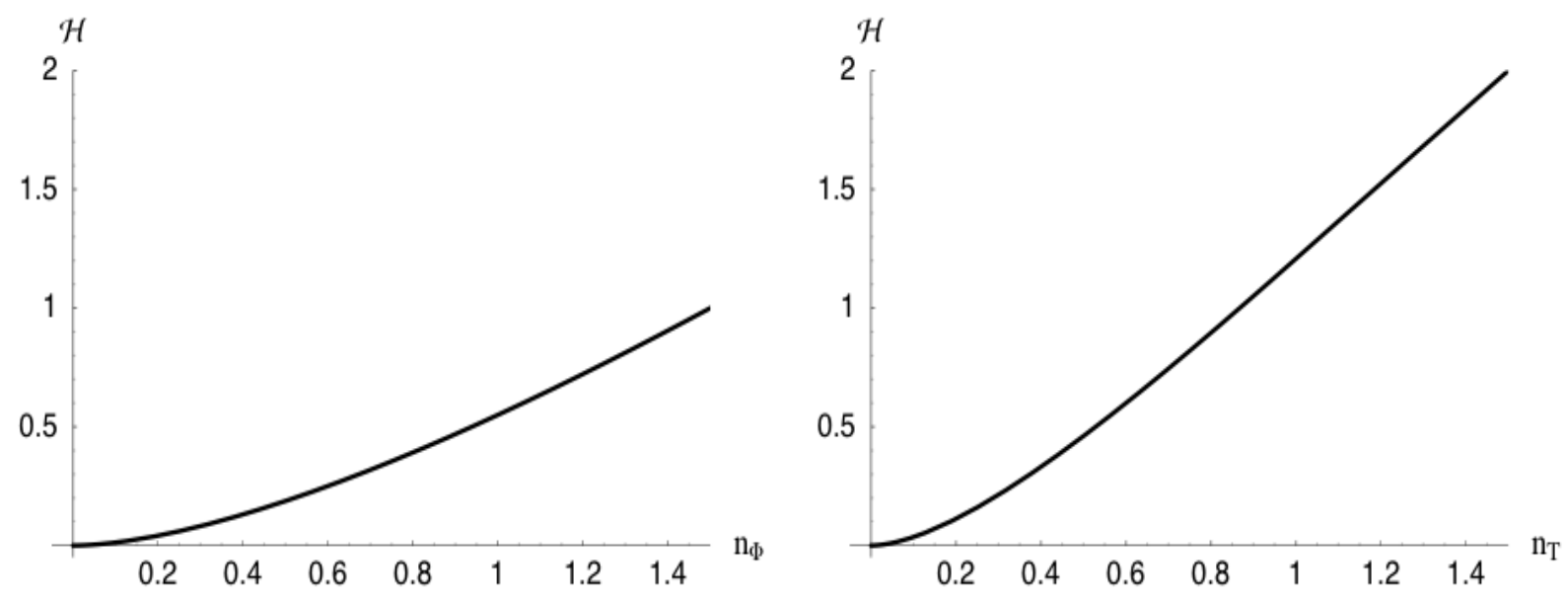

FIG. 2: On the left is the bound on the EOF based on a constrained $\hat{n}_{\Phi}$, Eq. (4.8). The plot on the right is the bound on the EOF based on a constrained negativity, Eq. 4.12).

and the bound can thus be extended to mixed states, giving

$$
h(\rho) \geq H_{2}\left(\frac{1+\sqrt{1-4 n_{\Phi}^{2} / 9}}{2}\right),
$$

with $n_{\Phi}$ being the $\Phi$-negativity of $\rho$.

The first step in bounding the EOF with only a single constraint on the negativity is to determine the function

$$
\widetilde{H}\left(n_{T}\right)=\min _{\boldsymbol{\mu}}\left\{H(\boldsymbol{\mu}) \mid \frac{\left(\sum_{j=1}^{4} \sqrt{\mu_{j}}\right)^{2}-1}{2}=n_{T}\right\}
$$

This was solved in [41, 33] for 2 or 3 Schmidt coefficients and recently shown to be valid for any number of Schmidt coefficients [42]. In particular, for four Schmidt coefficients, the case of interest here, we obtain

$$
\widetilde{H}\left(n_{T}\right)=H_{2}(\gamma)+(1-\gamma) \log _{2} 3
$$

with

$$
\gamma=\frac{\left(\sqrt{2 n_{T}+1}+\sqrt{3\left(3-2 n_{T}\right)}\right)^{2}}{16}
$$

Unlike $\widetilde{H}\left(\hat{n}_{\Phi}\right), \widetilde{H}\left(n_{T}\right)$ is not convex over the entire range of $n_{T}$. It is, however, a monotonically increasing function of $n_{T}$. The actual bound on the EOF is thus the convex-roof extension of this function, co $\left[\widetilde{H}\left(n_{T}\right)\right]$, which is given as 33 .

$$
h(\rho) \geq \mathcal{H}\left(n_{T}\right) \equiv \operatorname{co}\left[\widetilde{H}\left(n_{T}\right)\right]= \begin{cases}H_{2}(\gamma)+(1-\gamma) \log _{2} 3, & n_{T} \in[0,1], \\ \left(n_{T}-\frac{3}{2}\right) \log _{2} 3+2, & n_{T} \in\left[1, \frac{3}{2}\right] .\end{cases}
$$




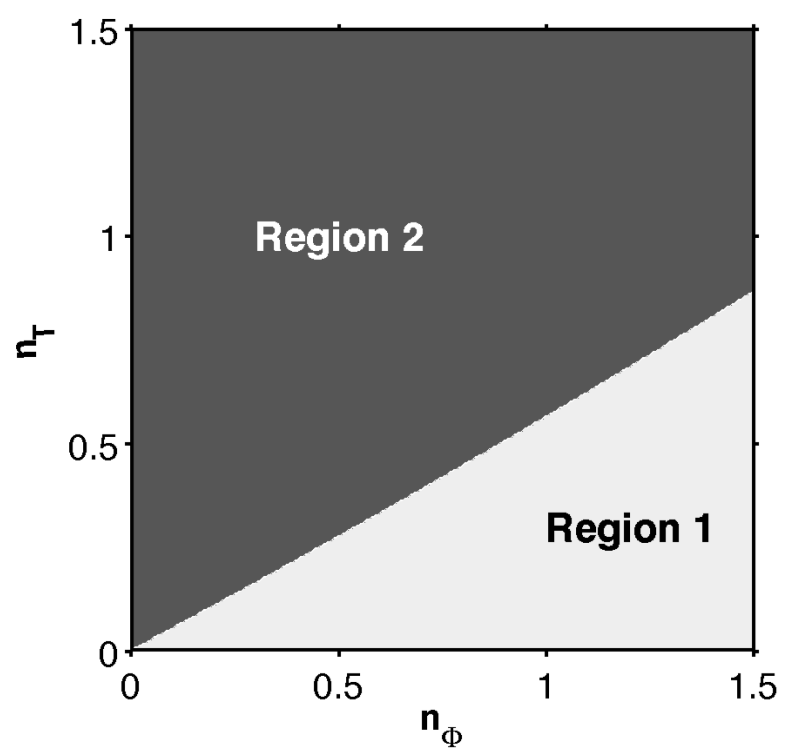

FIG. 3: In Region 1, the singly-constrained $n_{\Phi}$ bound is better than the singly-constrained $n_{T}$ bound. In Region 2, the opposite is true.

Both the singly constrained bounds are plotted in Fig. 2. It might seem that the bound based on the $\hat{n}_{\Phi}$ constraint is always poorer than that in Eq. 4.12), but this is not the case. There is a region in the $\hat{n}_{\Phi}-n_{T}$ plane where the bound of Eq. 4.8) is better than that of Eq. (4.12). This is depicted in Fig. 3 .

\section{B. Tangle and concurrence}

The procedure in the previous section can be undertaken for the tangle $t(\rho)$ and the concurrence $c(\rho)$ [16], [17]. To place bounds on the tangle, we start by finding

$$
\widetilde{T}\left(\hat{n}_{\Phi}\right)=\min _{\boldsymbol{\mu}}\left\{2\left(1-|\boldsymbol{\mu}|^{2}\right) \mid 3 \sqrt{\left(\mu_{1}+\mu_{4}\right)\left(\mu_{2}+\mu_{3}\right)}=\hat{n}_{\Phi}\right\},
$$

which gives a bound for pure states. Then, just as for the EOF, the bound on the tangle for mixed states is given by the convex hull of $\widetilde{T}\left(\hat{n}_{\Phi}\right)$,

$$
t(\rho) \geq \mathcal{T}\left(\hat{n}_{\Phi}\right) \equiv \operatorname{co}\left[\widetilde{T}\left(\hat{n}_{\Phi}\right)\right],
$$

provided $\widetilde{T}\left(\hat{n}_{\Phi}\right)$ is a monotonically nondecreasing function of $\hat{n}_{\Phi}$.

Using the normalization and $\hat{n}_{\Phi}$ constraints of Eq. (4.4), we have

$$
2\left(1-|\boldsymbol{\mu}|^{2}\right)=2\left(1-\sum_{i=1}^{4} \mu_{i}^{2}\right)=4 \sum_{i<j} \mu_{i} \mu_{j}=4\left(\frac{\hat{n}_{\Phi}^{2}}{9}+\mu_{1} \mu_{4}+\mu_{2} \mu_{3}\right) .
$$

Just as for the EOF, the minimization is trivial, the minimum occurring for the upper sign in Eq. (4.4), with $\mu_{4}=0\left(\mu_{1}=\alpha\right)$ and $\mu_{3}=0\left(\mu_{2}=\beta\right)$, thus giving

$$
\widetilde{T}\left(\hat{n}_{\Phi}\right)=\frac{4}{9} \hat{n}_{\Phi}^{2} .
$$


Since this is both monotonically increasing and convex in $\hat{n}_{\Phi}$, the same bound holds for mixed states, but in terms of the actual negativity $n_{\Phi}$, i.e.,

$$
t(\rho) \geq \mathcal{T}\left(n_{\Phi}\right)=\frac{4}{9} n_{\Phi}^{2} .
$$

The lower bound on the tangle, subject to a constraint on the negativity, is found by starting from

$$
\widetilde{T}\left(n_{T}\right)=\min _{\boldsymbol{\mu}}\left\{2\left(1-|\boldsymbol{\mu}|^{2}\right) \mid \frac{\left(\sum_{j=1}^{4} \sqrt{\mu_{j}}\right)^{2}-1}{2}=n_{T}\right\} .
$$

This is a relatively involved minimization, but it is exactly the same as the minimization problem that arises in evaluating a bound on the tangle for isotropic states, so we can adapt the result of [17] to give

$$
\widetilde{T}\left(n_{T}\right)=\frac{1}{12}\left(9+4 n_{T}^{2}+\sqrt{3\left(3+4 n_{T}-4 n_{T}^{2}\right)}\left(2 n_{T}-3\right)\right) .
$$

This quantity is monotonically increasing, but is not convex over the complete range of $n_{T}$. The convex hull $\mathcal{T}\left(n_{T}\right) \equiv \operatorname{co}\left[\widetilde{T}\left(n_{T}\right)\right]$ is required to extend the bound to mixed states. Again using the results of [17], we obtain

$$
t(\rho) \geq \mathcal{T}\left(n_{T}\right)= \begin{cases}\frac{1}{12}\left(9+4 n_{T}^{2}+\sqrt{3\left(3+4 n_{T}-4 n_{T}^{2}\right)}\left(2 n_{T}-3\right)\right), & n_{T} \in[0,1], \\ \frac{4}{3} n_{T}-\frac{1}{2}, & n_{T} \in\left[1, \frac{3}{2}\right] .\end{cases}
$$

We can derive from Eq. 4.17) an expression for the lower bound on the concurrence of $4 \times N$ states with a given value of $n_{\Phi}$ :

$$
c(\rho) \geq \mathcal{C}\left(n_{\Phi}\right)=\widetilde{C}\left(n_{\Phi}\right)=\sqrt{\widetilde{T}\left(n_{\Phi}\right)}=\frac{2}{3} n_{\Phi} .
$$

An expression for the minimum of the concurrence, subject to the negativity constraint, can be obtained from Eq. (4.19). The resulting function is everywhere concave, and thus its convex hull is a straight line joining the end points. This line is

$$
c(\rho) \geq \mathcal{C}\left(n_{T}\right)=\sqrt{\frac{2}{3}} n_{T} .
$$

The bounds on both the tangle and the concurrence are plotted in Fig 4. As was true for the EOF, the $n_{\Phi}$ bound is better than the $n_{T}$ bound in some parts of the $n_{\Phi}-n_{T}$ plane. This is shown in Fig. 5 .

Recently, a lower bound on the concurrence has been derived based on the negativity constraint [12], using techniques different from those employed here. That lower bound is exactly the one in Eq. (4.22). We have thus provided an independent derivation of the bound presented in [12. In addition, we can use the procedure from [12] to derive a lower bound on the tangle based on the $\hat{n}_{\Phi}$ constraint. Then we obtain

$$
\frac{\widetilde{T}\left(\hat{n}_{\Phi}\right)}{4}-\frac{\hat{n}_{\Phi}^{2}}{9}=\mu_{1} \mu_{4}+\mu_{2} \mu_{3} \geq 0
$$

which for general mixed states, leads exactly to the bound in Eq. (4.17). 

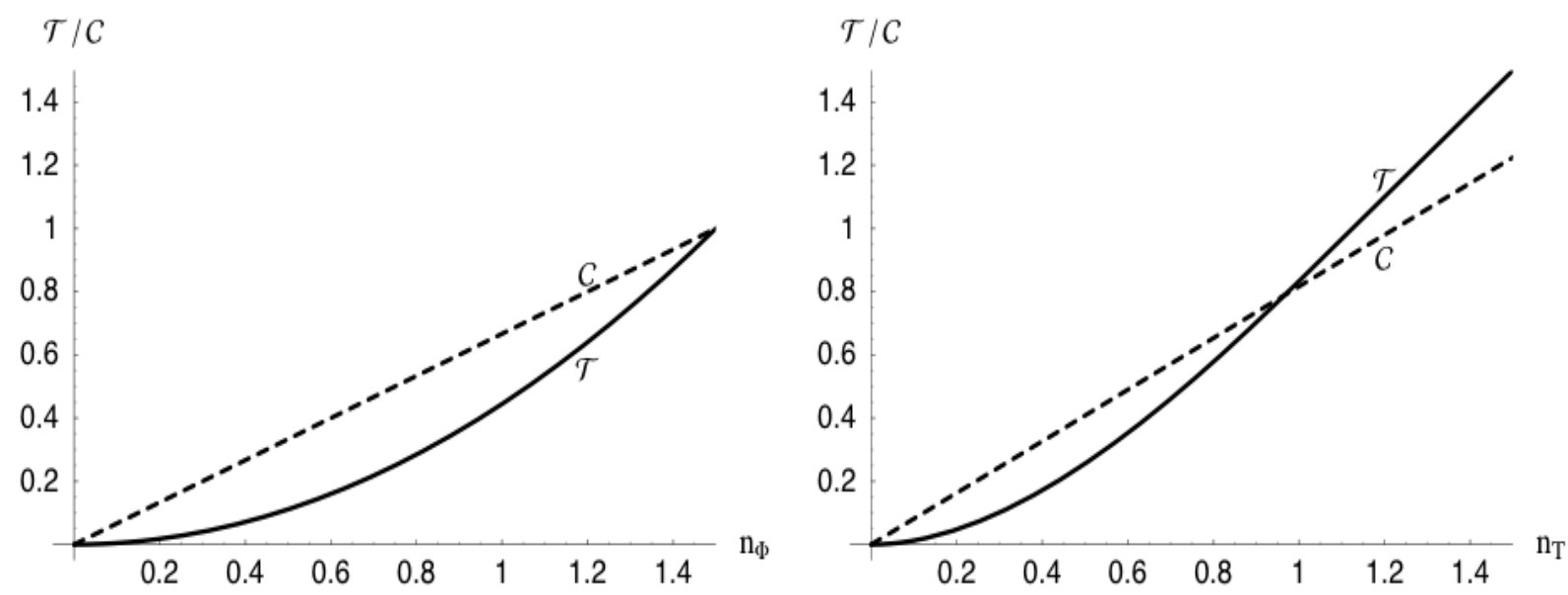

FIG. 4: The plot on the left shows the bounds on the tangle and the concurrence based on the $n_{\Phi}$ constraint. The solid line is the bound on the tangle and the dashed line is the bound on the concurrence. On the right is a plot of the analogous bounds based on the $n_{T}$ constraint.

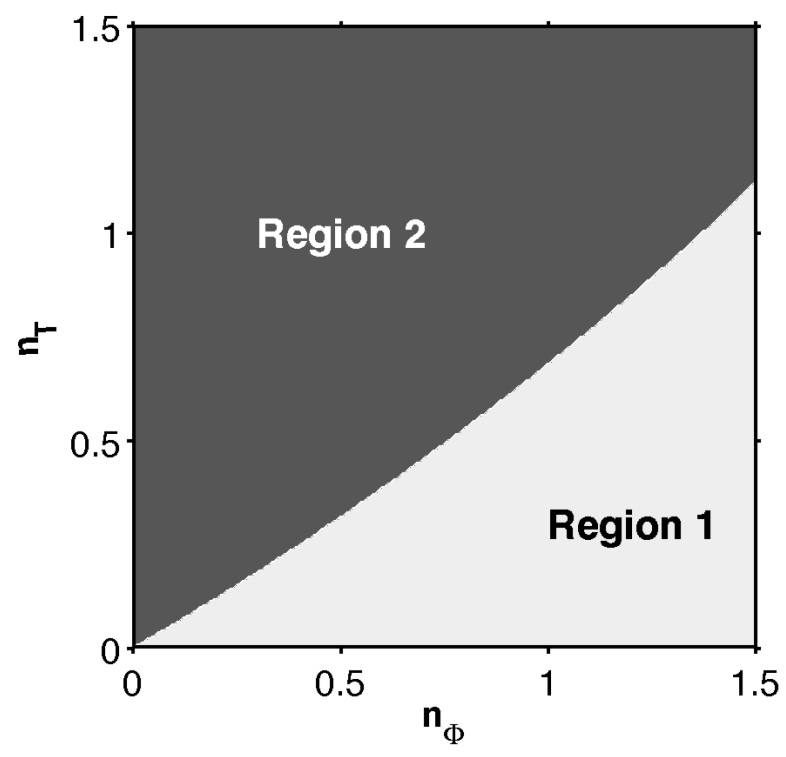

FIG. 5: Region 1 is where the $n_{\Phi}$ constraint is better than the $n_{T}$ constraint for bounding the tangle and concurrence. Region 2 is where the converse is true.

\section{DOUBLY CONSTRAINED BOUNDS}

In this section we place new lower bounds on the EOF, tangle, and concurrence for $4 \times N$ density operators by using $n_{T}$ and $\hat{n}_{\Phi}$ simultaneously as constraints. 


\section{A. Pure states of $4 \times N$ systems}

For a $4 \times N$ pure state, described by the Schmidt coefficients $\mu_{i}, i=1, \ldots, 4$, we have three constraint equations,

$$
\begin{aligned}
\frac{1}{2}\left[\left(\sqrt{\mu_{1}}+\sqrt{\mu_{2}}+\sqrt{\mu_{3}}+\sqrt{\mu_{4}}\right)^{2}-1\right] & =n_{T}, \\
3 \sqrt{\left(\mu_{1}+\mu_{4}\right)\left(\mu_{2}+\mu_{3}\right)} & =\hat{n}_{\Phi}, \\
\mu_{1}+\mu_{2}+\mu_{3}+\mu_{4} & =1,
\end{aligned}
$$

in addition to the inequality constraints

$$
1 \geq \mu_{1} \geq \mu_{2} \geq \mu_{3} \geq \mu_{4} \geq 0
$$

Both $\hat{n}_{\Phi}$ and $n_{T}$ take on values between 0 and $3 / 2$, so all $4 \times N$ states, pure or mixed, are mapped to a square of side $3 / 2$ in the $\hat{n}_{\Phi}-n_{T}$ plane. Not all points in the square correspond to pure states. If we solve the three equations in (5.1) simultaneously and express $\mu_{1}, \mu_{2}$ and $\mu_{3}$ in terms of $n_{T}, \hat{n}_{\Phi}$ and $\mu_{4}$ (see Appendix $\left(\mathrm{D}\right.$ ), we find that for some allowed values of $\hat{n}_{\Phi}$ and $n_{T}$, there is no allowed value of $\mu_{4}$ for which the other three Schmidt coefficients are real numbers between 0 and 1 in even one of the solution branches of (5.1).

To find the region occupied by pure states in the $\hat{n}_{\Phi}-n_{T}$ plane, let us use the pure-state expressions for $n_{T}$ and $\hat{n}_{\Phi}$ in Eq. (5.1) to find the largest and smallest values that $n_{T}$ can take on for a fixed value of $\hat{n}_{\Phi}$. We proceed as in the minimization of $H(\boldsymbol{\mu})$ in Sec. IV. Defining $\alpha=\mu_{1}+\mu_{4}$ and $\beta=\mu_{2}+\mu_{3}$, the normalization and $\hat{n}_{\Phi}$ constraints can be solved to give $\alpha$ and $\beta$ as in Eq. (4.4). The negativity takes the form

$$
\sqrt{2 n_{T}+1}=\sqrt{\mu_{1}}+\sqrt{\alpha-\mu_{1}}+\sqrt{\mu_{2}}+\sqrt{\beta-\mu_{2}} .
$$

It is trivial to see that the maximum of $n_{T}$ occurs when $\mu_{1}=\mu_{4}=\alpha / 2$ and $\mu_{2}=\mu_{3}=\beta / 2$. This maximum cannot be achieved, however, because we must respect the ordering $\mu_{1} \geq \mu_{2} \geq \mu_{3} \geq \mu_{4}$ that we assumed in our definition of $\hat{n}_{\Phi}$. We should always choose $\mu_{2}=\mu_{3}=\beta / 2$, but the best we can then do with $\mu_{1}$ and $\mu_{4}$ is to choose $\mu_{4}=\beta / 2, \mu_{1}=\alpha-\beta / 2$ when $\alpha \geq \beta$ [upper sign in Eq. 4.4] ] or $\mu_{1}=\beta / 2, \mu_{4}=\alpha-\beta / 2$ when $\beta \geq \alpha$ [lower sign in Eq. (4.4)]. The requirement that $\mu_{1} \leq \alpha$ implies that the latter case can only be used when $\hat{n}_{\Phi} \geq \sqrt{2}$. In both cases, the the maximum value of $n_{T}$ for fixed $\hat{n}_{\Phi}$ has the form

$$
n_{T}=\frac{1}{2}\left[(\sqrt{\alpha-\beta / 2}+3 \sqrt{\beta / 2})^{2}-1\right] .
$$

It turns out that the upper sign in Eq. (4.4) always gives a larger value for $n_{T}$. Using the upper sign, we find that the maximum of $n_{T}$ for fixed values of $\hat{n}_{\Phi}$ is given by

$$
n_{T}=\frac{3}{4}\left(1-\sqrt{1-\frac{4}{9} \hat{n}_{\Phi}^{2}}+\sqrt{\frac{4}{3} \hat{n}_{\Phi}^{2}+2 \sqrt{1-\frac{4}{9} \hat{n}_{\Phi}^{2}}-2}\right) .
$$


The minimum value of $n_{T}$ occurs on the boundary of allowed Schmidt coefficients, i.e., when $\mu_{1}=\alpha$ and $\mu_{2}=\beta$, with the upper sign in Eq. (4.4). Thus the minimum value of $n_{T}$ for a fixed value of $\hat{n}_{\Phi}$ is given by

$$
n_{T}=\frac{1}{2}\left[(\sqrt{\alpha}+\sqrt{\beta})^{2}-1\right]=\frac{1}{3} \hat{n}_{\Phi} .
$$

From Eqs. (5.4) and (5.6) we find that the pure states of a $4 \times N$ system lie in the region shown in Fig 6. Notice that for this case of two constraints, the pure-state region is not convex.

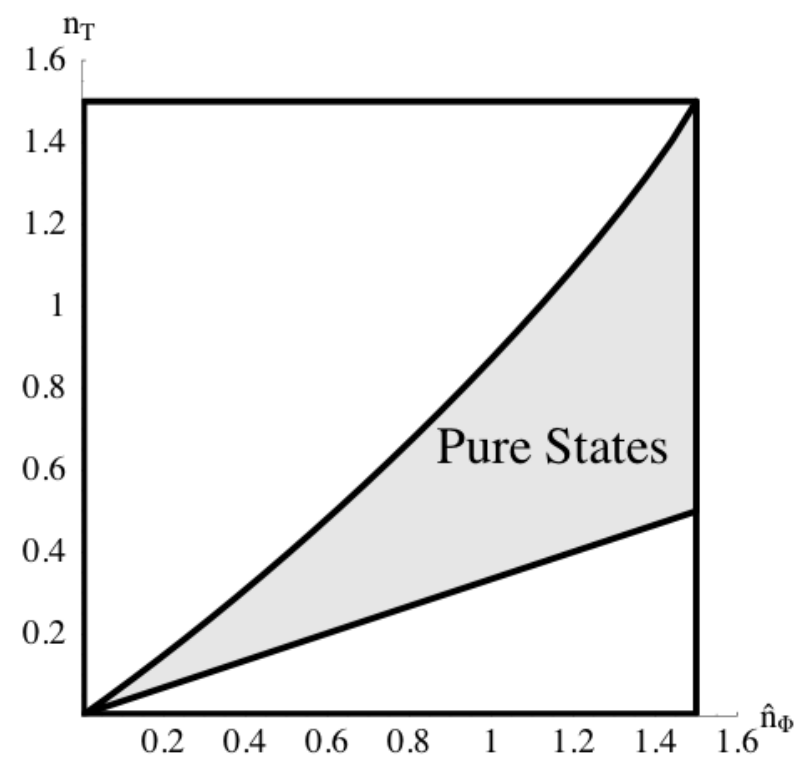

FIG. 6: The pure-state region in the $\hat{n}_{\Phi}-n_{T}$ plane for $4 \times N$ systems.

\section{B. Entanglement of formation}

The EOF for pure bipartite states is a concave function of the marginal density operator obtained by tracing over one of the subsystems. This means that it is a concave function of the Schmidt coefficients $\boldsymbol{\mu}$. Searching for a minimum is not the most natural thing one can do with a concave function, yet this is what we are instructed to do by the procedure for bounding the EOF outlined in Sec. II B. Starting from the EOF $H(\boldsymbol{\mu})$ for pure bipartite $4 \times N$ states, our objective is to find a convex, monotonic function $\mathcal{H}(\mathbf{n})$ as outlined in the Sec. II. This function will be our lower bound on the EOF for all states.

The first step is to find the function

$$
\widetilde{H}(\mathbf{n})=\widetilde{H}\left(\hat{n}_{\Phi}, n_{T}\right) \equiv \min _{\boldsymbol{\mu}}\left\{H(\boldsymbol{\mu}) \mid 3 \sqrt{\left(\mu_{1}+\mu_{4}\right)\left(\mu_{2}+\mu_{3}\right)}=\hat{n}_{\Phi}, \frac{\left(\sum_{j} \sqrt{\mu_{j}}\right)^{2}-1}{2}=n_{T}\right\},
$$

which is defined on the pure-state region. The method of Lagrange multipliers is not suitable for finding the minimum in Eq. (5.7) because the problem is over-constrained. The equations that we 
obtain using Lagrange multipliers have a consistent solution only if $\hat{n}_{\Phi}$ and $n_{T}$ are related as in Eq. (5.5) and therefore lie on the upper boundary of the pure-state region. This does not mean that there is no minimum for $H(\boldsymbol{\mu})$, but rather that the minimum lies on a boundary of the allowed values of $\boldsymbol{\mu}$.

We already know $\widetilde{H}\left(\hat{n}_{\Phi}, n_{T}\right)$ on the boundaries of the pure-state region. The boundary with three of the Schmidt coefficients being zero is the origin in the $\hat{n}_{\Phi} n_{T}$ plane where $\widetilde{H}\left(\hat{n}_{\Phi}, n_{T}\right)=$ $H(\boldsymbol{\mu})=0$. The boundary with two of the Schmidt coefficients zero lies on the line $n_{T}=\hat{n}_{\Phi} / 3$. To find the value of $\widetilde{H}\left(\hat{n}_{\Phi}, n_{T}\right)$ along this boundary, note that the minimum of the EOF subject to just the $\hat{n}_{\Phi}$ constraint occurs for $\boldsymbol{\mu}_{\Phi}=(\alpha, 1-\alpha, 0,0)$, where $\alpha$ is given in Eq. (4.4). Substituting $\boldsymbol{\mu}_{\Phi}$ into $n_{T}$ we get $n_{T}=\sqrt{\alpha(1-\alpha)}=\hat{n}_{\Phi} / 3$. This means that along the line $n_{T}=\hat{n}_{\Phi} / 3$, the $n_{T}$ constraint is automatically satisfied if the $\hat{n}_{\Phi}$ constraint is satisfied. Thus along the lower boundary of the pure-state region, we have $\widetilde{H}\left(\hat{n}_{\Phi}, n_{T}\right)=\widetilde{H}\left(\hat{n}_{\Phi}\right)$. Similarly, along the upper boundary of the pure-state region, the $\hat{n}_{\Phi}$ constraint comes for free. This is because the minimum of the EOF subject to the $n_{T}$ constraint occurs when the Schmidt coefficients are given by $\boldsymbol{\mu}_{T}=\left(\gamma, \gamma^{\prime}, \gamma^{\prime}, \gamma^{\prime}\right)$ with $\gamma$ given by Eq. (4.11) and $\gamma^{\prime}=(1-\gamma) / 3$. The doubly-constrained problem reduces to the singly-constrained problem when $\hat{n}_{\Phi}=\sqrt{2(2 \gamma+1)(1-\gamma)}$. Relabelling $\gamma$ as $\alpha-\beta / 2$ and $\gamma^{\prime}$ as $\beta / 2$ we see that the $\hat{n}_{\Phi}$ constraint is automatically satisfied along the upper boundary of the pure-state region if the $n_{T}$ constraint is satisfied. Hence along the upper boundary of the pure-state region, we have $\widetilde{H}\left(\hat{n}_{\Phi}, n_{T}\right)=\widetilde{H}\left(n_{T}\right)$.

These considerations mean that for the entanglement of formation, the monotone boundaries that we define in Appendix A coincide with the boundaries of the pure-state region, making it unnecessary to construct the monotonically nondecreasing function $\widetilde{H}_{\uparrow}\left(\hat{n}_{\Phi}, n_{T}\right)$, since $\widetilde{H}\left(\hat{n}_{\Phi}, n_{T}\right)$ is itself monotonically nondecreasing.

The minimum of $H(\boldsymbol{\mu})$ in the remaining part of the pure-state region can be found using the straightforward numerical procedure described below. We start from the two distinct sets of solutions $\boldsymbol{\mu}^{(1)}$ and $\boldsymbol{\mu}^{(2)}$ of the three constraint equations (see Appendix D). We go to the boundary where one of the Schmidt coefficients is zero by setting $\mu_{4}=0$ in the solutions. Now compute $H\left(\boldsymbol{\mu}^{(1)}\right)$ and $H\left(\boldsymbol{\mu}^{(2)}\right)$ corresponding to the two solutions in the regions in the $\hat{n}_{\Phi}-n_{T}$ plane where each of the solutions is valid. The solutions are not valid in the whole pure-state region because the three Schmidt coefficients have to be real, nonnegative numbers less than one. All points in the pure-state region cannot be covered if we set $\mu_{4}=0$. This is easily seen by noticing that the point $\hat{n}_{\Phi}=n_{T}=3 / 2$ corresponds to the fully entangled $4 \times N$ state and for this state all four Schmidt coefficients have the value $1 / 4$. The fully entangled state and other states close to it cannot be reached using the procedure described above if we stay on the boundary defined by $\mu_{4}=0$. So we start increasing the value of $\mu_{4}$ in small steps until it reaches $1 / 4$. The parts of the 2 -constraint region that are covered by different choices of $\mu_{4}$ are shown in Fig. 7.

This numerical procedure gives us ranges of values of $\mu_{4}$ over which $H\left(\boldsymbol{\mu}^{(1)}\right)$ and/or $H\left(\boldsymbol{\mu}^{(2)}\right)$ can be calculated at each point in the pure-state region. For the value of $\widetilde{H}(\mathbf{n})$ at each point, we pick the minimum over the allowed range of values for $\mu_{4}$ at that point.

The function $\widetilde{H}(\mathbf{n})$ in the pure-state region is shown in Fig. 8. It is, as required, a monotonically increasing function of both $\hat{n}_{\Phi}$ and $n_{T}$. Along the upper boundary of the pure-state region, the 

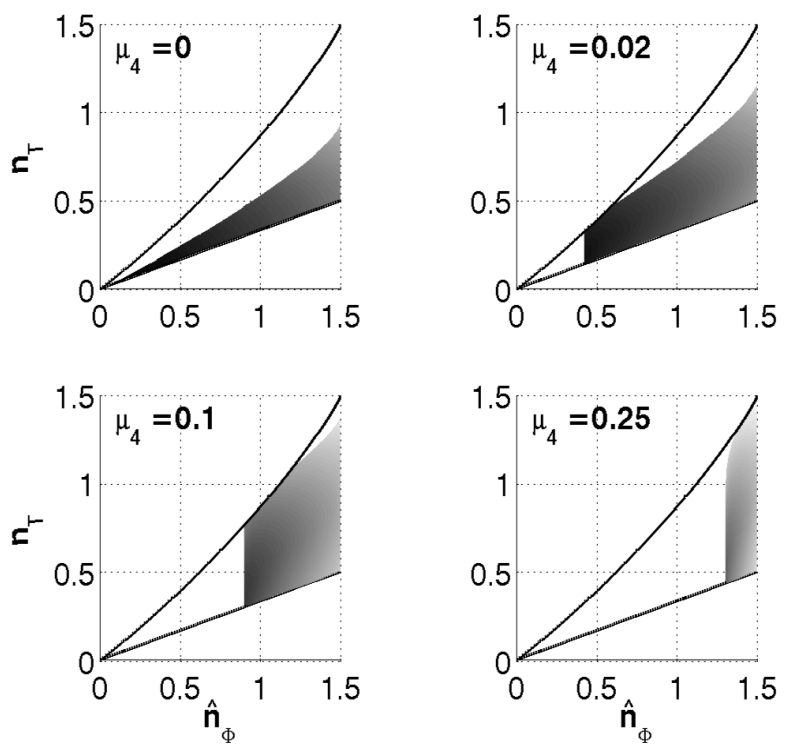

FIG. 7: The part of the 2-constraint region in which a value for $\widetilde{H}(\mathbf{n})$ can be computed is shown for four values of $\mu_{4}=0,0.02,0.1$, and 0.25 . The two lines are the boundaries of the pure-state region.
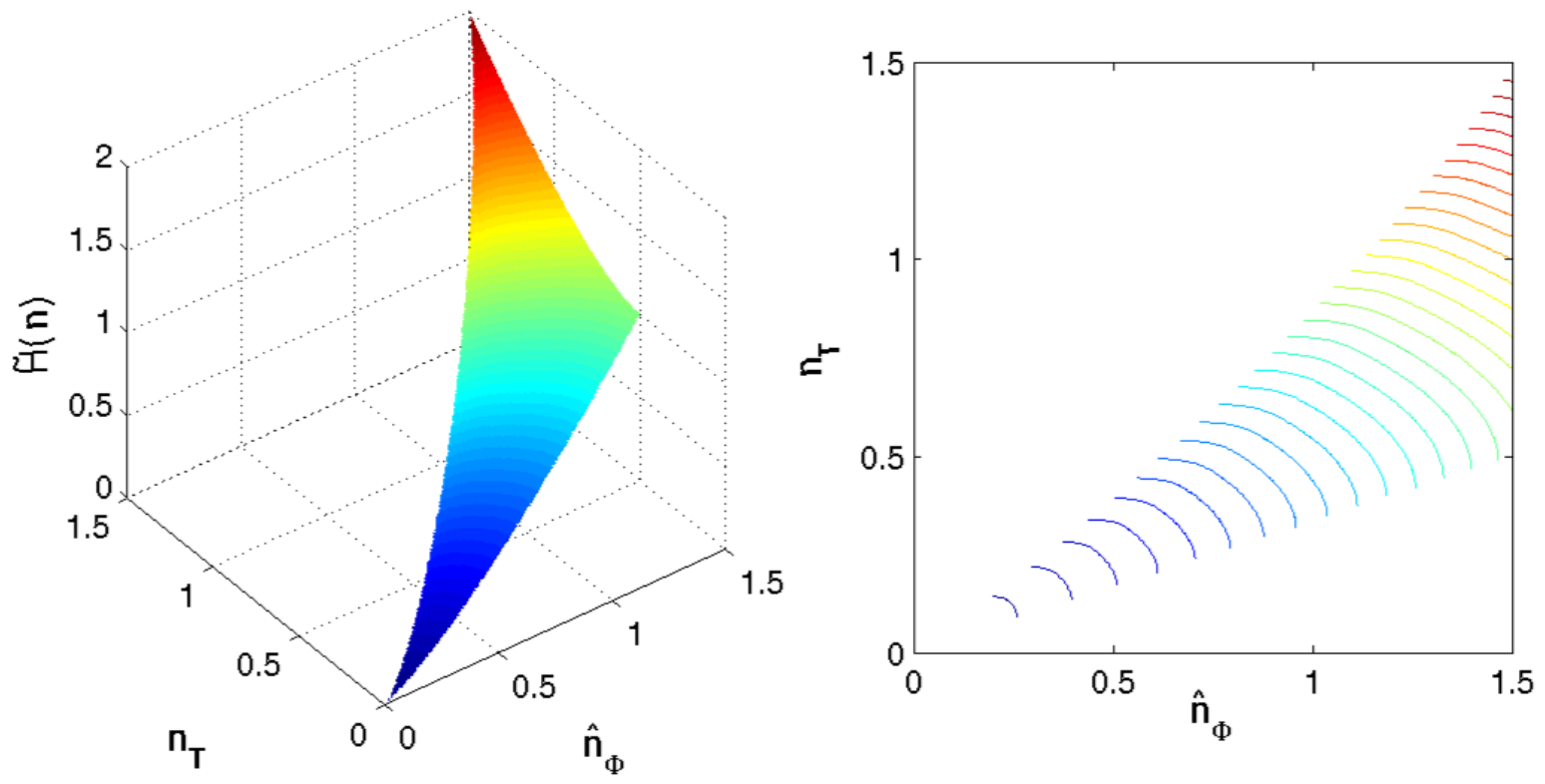

FIG. 8: (Color online) Plots of $\widetilde{H}(\mathbf{n})$, the minimum of the entropy of formation, $H(\boldsymbol{\mu})$, in the pure-state region. On the left side is a 3-dimensional plot of $\widetilde{H}(\mathbf{n})$ and on the right is a contour plot of the same function.

numerically computed value of $\widetilde{H}(\mathbf{n})$ matches the value of $\widetilde{H}\left(n_{T}\right)$ from Eq. 4.9 . In addition to this, from the contour plot of $\widetilde{H}(\mathbf{n})$ in Fig. 8, we see that along the upper boundary, the function has zero slope along the $\hat{n}_{\Phi}$ direction.

The function $\widetilde{H}(\mathbf{n})$ is not convex, which can be seen by computing the Hessian at every point in the pure-state region. If the function were convex, both eigenvalues of the Hessian would be 
positive at all points. It turns out that one of the eigenvalues of the Hessian is negative in a region in the upper right corner of the $\hat{n}_{\Phi}-n_{T}$ plane, close to the maximally entangled state.

Since $\widetilde{H}(\mathbf{n})$ is not convex, we have to compute its convex hull,

$$
\mathcal{H}(\mathbf{n})=\operatorname{co}[\widetilde{H}(\mathbf{n})]
$$

to obtain the bound on the EOF in the pure-state region. The convex hull of $\widetilde{H}(\mathbf{n})$ can be computed numerically, and it turns out that the difference between $\mathcal{H}(\mathbf{n})$ and $\widetilde{H}(\mathbf{n})$ is quite small $\left(\sim 10^{-3}\right)$, the two differing differ only in a small region in the upper right corner of the pure-state region. As shown in Appendix B, taking the convex hull preserves monotonicity.
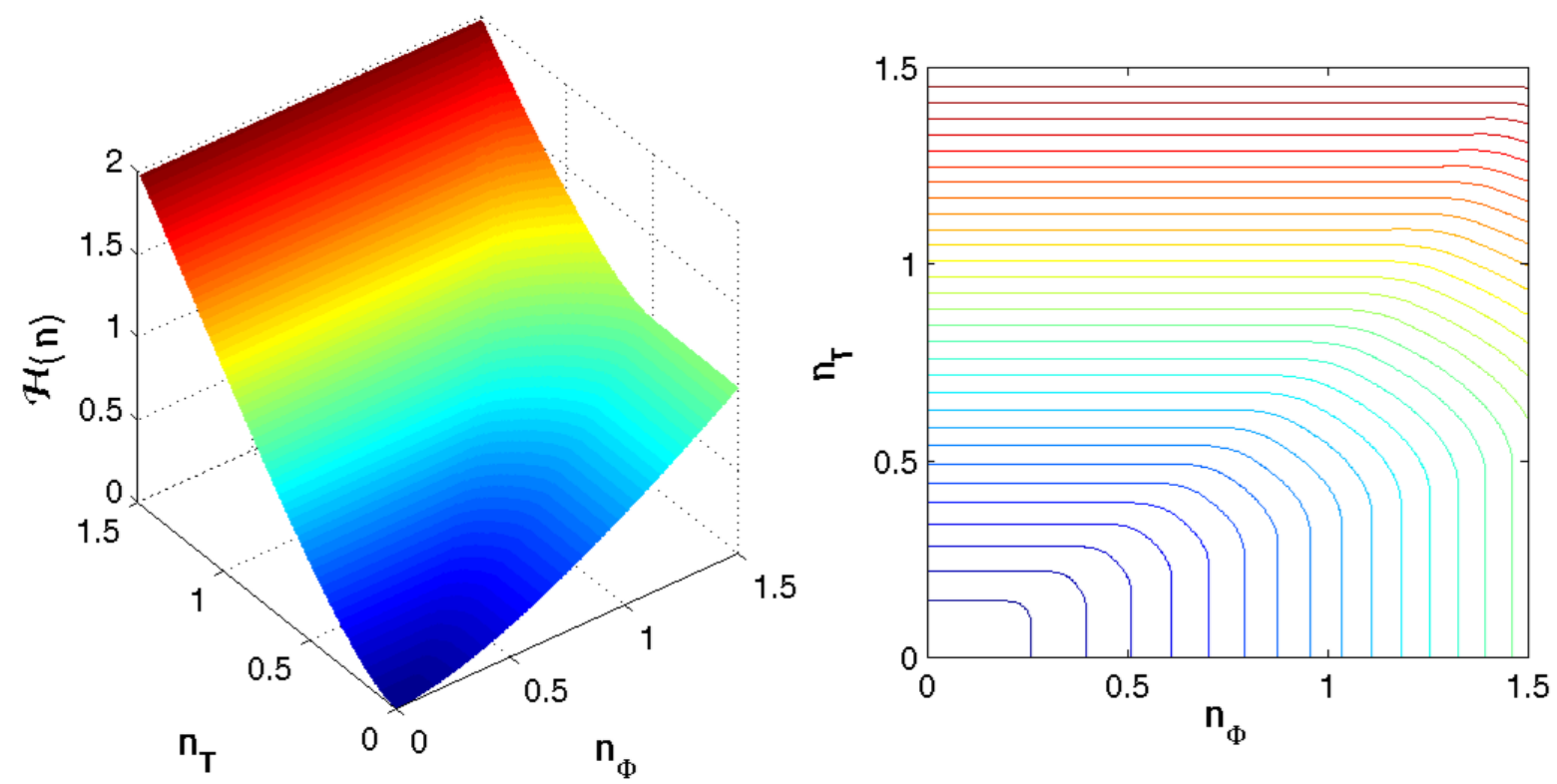

FIG. 9: (Color online) The doubly-constrained bound $\mathcal{H}(\mathbf{n})$ on the EOF of all $4 \times N$ states. On the right side is a contour plot of the same function.

To obtain a bound on the EOF of all $4 \times N$ states, we have to extend $\mathcal{H}(\mathbf{n})$ out of the pure-state region to the rest of the $\hat{n}_{\Phi}-n_{T}$ plane. The extension has to respect the monotonicity of $\mathcal{H}(\mathbf{n})$ so that the string of inequalities Eq. 2.8 holds. This can be achieved by extending $\mathcal{H}(\mathbf{n})$ using surfaces that match the function at the lower and upper boundaries of the pure-state region. To preserve monotonicity, the surface added on to the region below the lower boundary of the set of pure states has zero slope along the $n_{T}$ direction, and the surface added on to the region above the upper boundary of the set of pure states has zero slope along the $\hat{n}_{\Phi}$ direction. The resulting doubly-constrained bound $\mathcal{H}(\mathbf{n})$ on the EOF is shown in Fig. 9. We see from the figure that the extension to the whole $n_{\Phi}-n_{T}$ plane produces a smooth and seamless surface.

One final point worth mentioning involves the use of our bound for general mixed states. To do so, one must calculate $n_{\Phi}$ for the mixed state, and this calculation depends on the choice of an angular-momentum basis for system $B$ in order to define the $\Phi$-map. The bound itself thus depends on this choice of basis, and the best bound would generally be found for the basis choice 
that gives the largest value of $n_{\Phi}$. For pure states, for example, the results in Fig. 1 show that the best choice of basis is the Schmidt basis for system $B$.

The isotropic states, which lie along the diagonal in the $\hat{n}_{\Phi}-n_{T}$ plane, are special in that they saturate the singly-constrained bound $\mathcal{H}\left(n_{T}\right)$ from Eq. 4.12 . These states thus furnish a good consistency test of our doubly-constrained bound because our bound must match the singly-constrained bound when applied to isotropic states. A comparison of the two bounds for isotropic states is given in Fig. 10 .

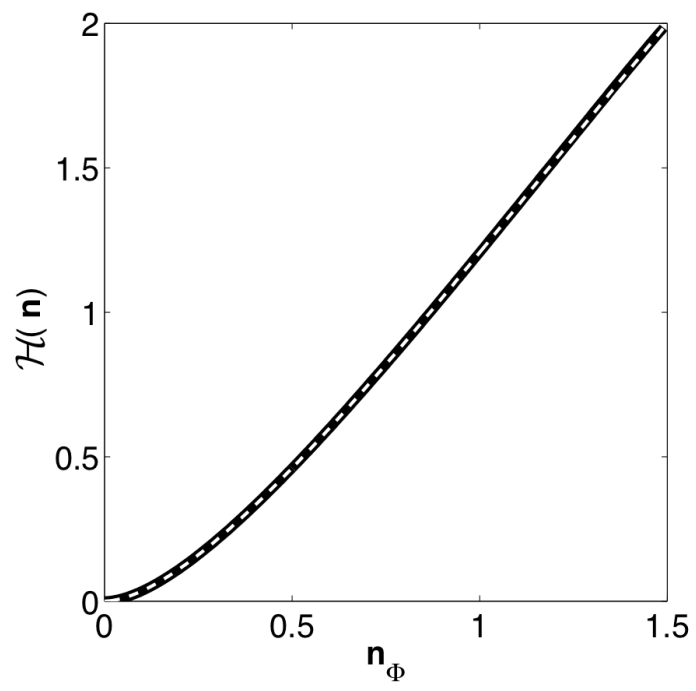

FIG. 10: The thick black line is the doubly-constrained bound on the EOF for isotropic states. The dashed white line, lying on top of the black line, is the singly-constrained bound $\mathcal{H}\left(n_{T}\right)$ from Eq. 4.12 .

We can make a second comparison between the singly and doubly constrained bounds using Fig. 10. From the way we constructed $\mathcal{H}(\mathbf{n})$, we know that its value on the diagonal in the $\hat{n}_{\Phi^{-}} n_{T}$ plane is the same as its value on the upper boundary of the pure-state region. We also know that the upper boundary is where the singly-constrained bound and the doubly-constrained bound are the same. From Fig. 10 , we see that the convex hull $\mathcal{H}\left(n_{T}\right)$ of the function $\widetilde{H}\left(n_{T}\right)$ of one variable matches the convex hull $\mathcal{H}(\mathbf{n})$ of the function $\widetilde{H}(\mathbf{n})$ of two variables on the upper pure-state boundary. These consistency checks give us increased confidence in the accuracy of our results.

\section{Tangle and concurrence}

Doubly-constrained bounds can be placed on the tangle and the concurrence of $4 \times N$ states by extending the procedure used for the EOF. For the tangle, we start by finding the function,

$$
\widetilde{T}(\mathbf{n})=\min _{\boldsymbol{\mu}}\left\{2\left(1-|\boldsymbol{\mu}|^{2}\right) \mid 3 \sqrt{\left(\mu_{1}+\mu_{4}\right)\left(\mu_{2}+\mu_{3}\right)}=\hat{n}_{\Phi}, \frac{\left(\sum_{j} \sqrt{\mu_{j}}\right)^{2}-1}{2}=n_{T}\right\},
$$

in the pure-state region. For the concurrence, we want the function $\widetilde{C}(\mathbf{n})=\sqrt{\widetilde{T}(\mathbf{n})}$, since for pure states the concurrence is the square root of the tangle. 

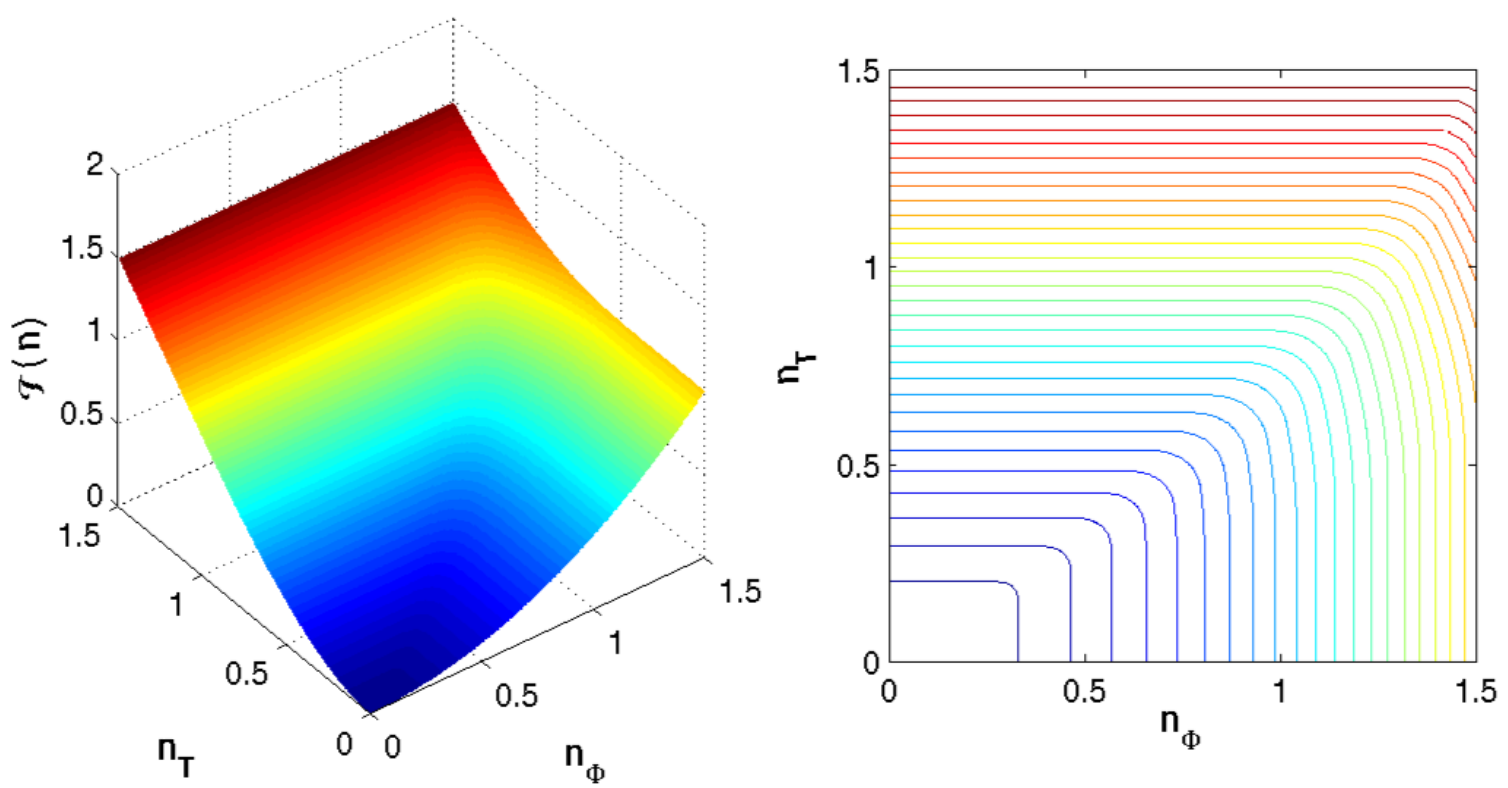

FIG. 11: (Color online) The doubly-constrained bound $\mathcal{T}(\mathbf{n})$ on the tangle of $4 \times N$ states. On the right side is a contour plot of the same function.
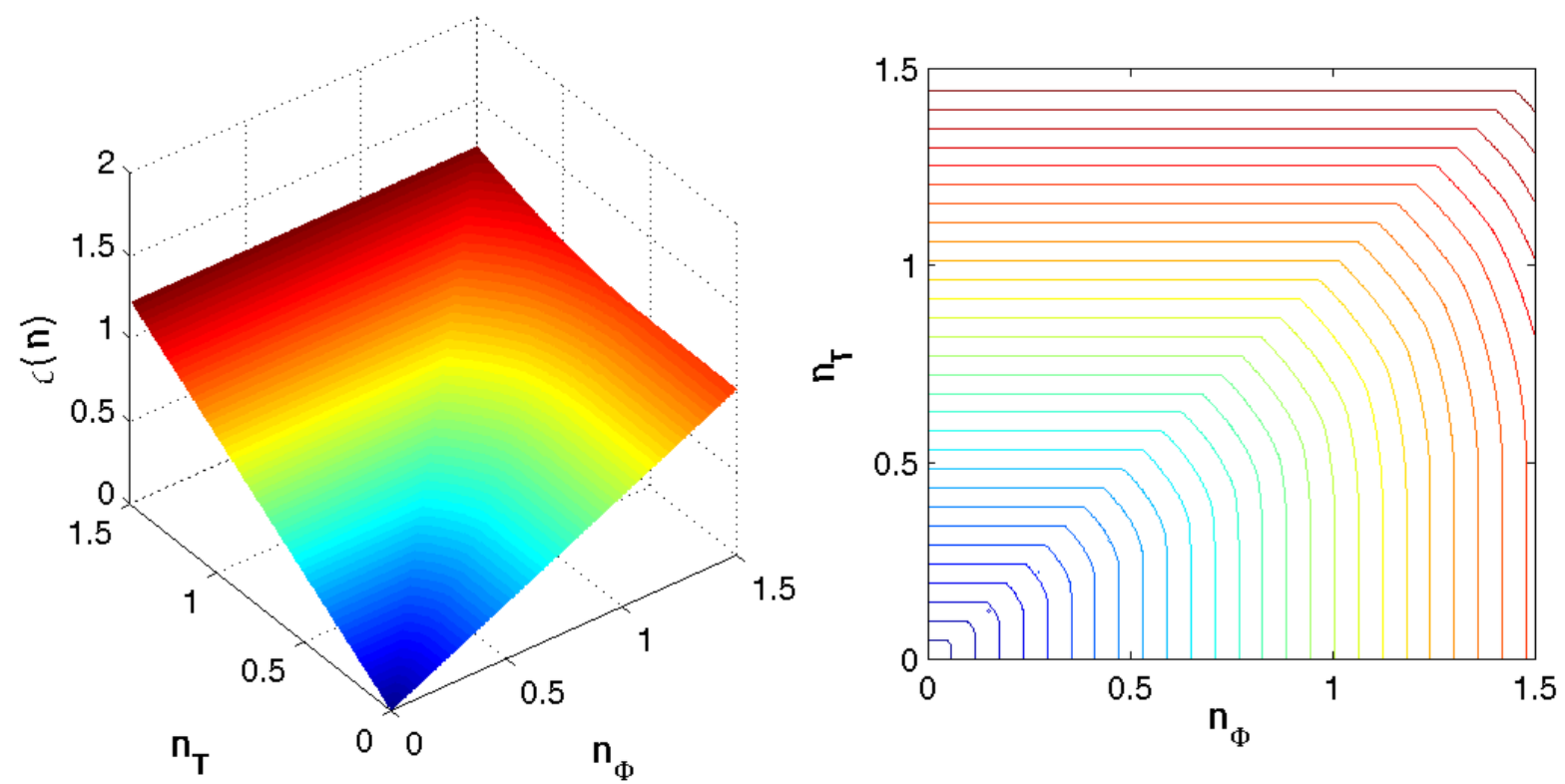

FIG. 12: (Color online) The doubly-constrained bound $\mathcal{C}(\mathbf{n})$ on the concurrence of $4 \times N$ states. On the right side is a contour plot of the same function.

For all three of the entanglement monotones, EOF, tangle and concurrence, the monotone boundaries we define in Appendix $\mathrm{A}$ coincide with the boundaries of the pure-state region. This is because the singly-constrained bounds for all three measures correspond to the same sets of Schmidt coefficients, $\boldsymbol{\mu}_{T}=\left(\gamma, \gamma^{\prime}, \gamma^{\prime}, \gamma^{\prime}\right)$ and $\boldsymbol{\mu}_{\Phi}=(\alpha, 1-\alpha, 0,0)$, and we have already seen for the EOF that these Schmidt coefficients define the boundaries of the pure-state region. This makes 
it unnecessary for these entanglement monotones to construct the monotonically nondecreasing function discussed in Appendix A. In general, for two different measures of entanglement and two constraints, the singly-constrained bounds for the two measures need not correspond to the same Schmidt coefficients.

Once we have $\widetilde{T}(\mathbf{n})$, the convex hull of this function extended to the whole $\hat{n}_{\Phi}-n_{T}$ plane is the doubly-constrained bound on the tangle, $\mathcal{T}(\mathbf{n})$. A three-dimensional plot and a contour plot of $\mathcal{T}(\mathbf{n})$ are shown in Fig. 11 .

The bound on the concurrence is the convex hull of the surface obtained from $\widetilde{C}(\mathbf{n})$. The resulting bound on the concurrence, $\mathcal{C}(\mathbf{n})$, is shown in Fig. 12 .

\section{CONCLUSION}

We focused on two aspects of the problem of quantifying entanglement in this paper. The first was a comparison between the bounds on different measures of entanglement obtained by using $n_{T}$ and $\hat{n}_{\Phi}$ independently as constraints. The second was the construction of doubly-constrained bounds on the three measures of entanglement that we considered.

Starting from the $\Phi$-map [11], we found that we can define an entanglement measure, which we call the $\Phi$-negativity. The $\Phi$-negativity of arbitrary quantum states can be calculated in a straightforward manner, just like their negativity. We also found that we can obtain a much simpler function $\hat{n}_{\Phi}$ of the Schmidt coefficients of pure states that is an upper bound on their $\Phi$-negativity. Previous work [33, [12] has shown that the negativity can be used as a constraint to place bounds on the EOF, the tangle, and the concurrence of bipartite states. We obtained a different set of bounds on these three measures of entanglement for $4 \times N$ mixed states by using $\hat{n}_{\Phi}$ instead as the constraint. The scheme for placing lower bounds on nonoperational measures of entanglement is general enough to allow us to use $\hat{n}_{\Phi}$ instead of $n_{\Phi}$ as the constraint. We were then able to compare the two sets of bounds on the measures of entanglement coming from using either one of the two operational entanglement measures as a single constraint.

We found that the $\hat{n}_{\Phi} n_{T}$ plane for pure states can be divided into two regions depending on which constraint led to the better bound on a given measure of entanglement. This prompted us to consider whether we can construct a single, composite bound for each measure of entanglement, applicable to the entire $\hat{n}_{\Phi}-n_{T}$ plane, by using both constraints simultaneously. It turned out that for $4 \times N$ systems this is a tractable problem, and we obtained doubly-constrained lower bounds for the first time for the EOF, the tangle, and the concurrence. We showed how the bounds on the different measures of entanglement obtained for pure states can be extended to include all states. We found that the requirement of monotonicity on the bound defined on pure states dictates how to extend the bound to all states. 


\section{APPENDIX A: GENERAL CONSTRUCTION OF $\widetilde{G}_{\uparrow}(\mathbf{n})$.}

In this Appendix, we describe the general procedure for constructing the monotonically nondecreasing function $\widetilde{G}_{\uparrow}(\mathbf{n})$, which replaces $\widetilde{G}(\mathbf{n})$ when the latter function is not itself monotonically nondecreasing.

As mentioned in Sec IIB, pure states of the system correspond to a simply connected subset, called the pure-state region, in the state hypercube in $\mathbb{R}^{K}$; the function $\widetilde{G}(\mathbf{n})$ is defined only on the pure-state region. Within the pure-state region, we can define $K$ hypersurfaces $\mathcal{S}_{k}$ as those on which the $k$ th constraint equation, $F_{k}(\boldsymbol{\mu})=n_{k}$, is automatically satisfied if the remaining $K-1$ constraint equations are satisfied. We denote the value of $n_{k}$ on $\mathcal{S}_{k}$ by $n_{k}^{*}\left(\mathbf{n}^{\prime}\right)$ where $\mathbf{n}^{\prime}=$ $\left(n_{1}, \ldots, n_{k-1}, n_{k+1}, \ldots, n_{K}\right)$; the function $n_{k}^{*}\left(\mathbf{n}^{\prime}\right)$ can be regarded as the defining equation for $S_{k}$. On the hypersurfaces $S_{k}, \widetilde{G}(\mathbf{n})$ is effectively defined by $K-1$ constraints. We denote the value of $\widetilde{G}(\mathbf{n})$ on $\mathcal{S}_{k}$ by $\widetilde{G}_{k}\left(\mathbf{n}^{\prime}\right)$. The minimum of any function subject to $K$ constraints is always greater than or equal to its value when subject to $K-1$ of these constraints, so we have $\widetilde{G}\left(\mathbf{n}^{\prime}, n_{k}\right) \geq \widetilde{G}_{k}\left(\mathbf{n}^{\prime}\right)$, where we have let $\left(\mathbf{n}^{\prime}, n_{k}\right) \equiv \mathbf{n}$. The inequality is saturated when $n_{k}=n_{k}^{*}\left(\mathbf{n}^{\prime}\right)$. Now consider $\widetilde{G}\left(\mathbf{n}^{\prime}, n_{k}\right)$ as a function of $n_{k}$. If we fix $\mathbf{n}^{\prime}$ and increase $n_{k}$, starting from its lowest value, then $\widetilde{G}\left(\mathbf{n}^{\prime}, n_{k}\right)$ has to either decrease or remain constant until we cross the hypersurface $\mathcal{S}_{k}$. For $n_{k} \geq n_{k}^{*}\left(\mathbf{n}^{\prime}\right), \widetilde{G}\left(\mathbf{n}^{\prime}, n_{k}\right)$ is a nondecreasing function of $n_{k}$. We want $\widetilde{G}_{\uparrow}(\mathbf{n})$ to be a nondecreasing function for all $n_{k}$, so we define it by

$$
\widetilde{G}_{\uparrow}\left(\mathbf{n}^{\prime}, n_{k}\right)=\left\{\begin{array}{cc}
\widetilde{G}_{k}\left(\mathbf{n}^{\prime}\right) & n_{k} \leq n_{k}^{*}\left(\mathbf{n}^{\prime}\right) \\
\widetilde{G}(\mathbf{n}) & n_{k}>n_{k}^{*}\left(\mathbf{n}^{\prime}\right),
\end{array} \quad k=1, \ldots, K .\right.
$$

The construction of $\widetilde{G}_{\uparrow}(\mathbf{n})$ is not complete at this point. Within each $(K-1)$-dimensional hypersurface, we will encounter $(K-2)$-dimensional hypersurfaces where two of the constraints are automatically satisfied. Across each of these $(K-2)$-dimensional hypersurfaces, we can update the value of $\widetilde{G}_{\uparrow}(\mathbf{n})$ just as described above.

There can be at most $K$ different $(K-1)$-constraint regions and the $K$-constraint region will, in general, be surrounded by these $(K-1)$-constraint regions. The $(K-1)$-constraint regions are surrounded, in turn, by $(K-2)$-constraint regions and so on. This construction procedure evidently terminates after $K-1$ steps. We call the hypersurfaces identified in this appendix monotone boundaries, because the nested structure of $k$-constraint regions they define are the key to constructing the monotonically nondecreasing function $\widetilde{G}_{\uparrow}(\mathbf{n})$ from $\widetilde{G}(\mathbf{n})$. In the examples we consider in Sec. V, the monotone boundaries coincide with the boundaries of the pure-state region, so we do not have to construct the function $\widetilde{G}_{\uparrow}(\mathbf{n})$.

\section{APPENDIX B: THE CONVEX HULL AND MONOTONICITY}

Here we show that the convex hull of a monotonically nondecreasing function on $\mathbb{R}^{K}$ is also monotonically nondecreasing. We first define a partial order on the set of vectors in $\mathbb{R}^{K}$ by defining $\mathbf{x} \geq \mathbf{y}$ to mean $x_{k} \geq y_{k}$ for all $k$. Define a monotone to be a function $f: \mathcal{D} \mapsto[0,1]$ satisfying the following conditions: 
1. The domain $\mathcal{D}$ is a bounded region contained in the positive orthant (including boundaries) of $\mathbb{R}^{K}$,

2. $0 \in \mathcal{D}$ and $f(0)=0$,

3. $\forall \mathbf{x}, \mathbf{y} \in \mathcal{D}$, if $\mathbf{x} \geq \mathbf{y}$, then $f(\mathbf{x}) \geq f(\mathbf{y}) . \quad$ (monotonicity)

The function $f$ can alternatively be viewed as a set of points in $\mathbb{R}^{K+1}$ given by the tuples $\left(x_{1}, \ldots, x_{K}, f(\mathbf{x})\right)$. Viewed this way, we can define the convex hull $C$ of $f$ as a set to be the smallest convex set containing the set $f$. We can also define the function $c: \mathcal{D}^{\prime} \mapsto[0,1]$, to be the convex hull of $f$ as a function. Thus $c$ is the largest convex function bounded from above by $f$; in this paper $c$ is called the convex roof of $f$; Clearly, $c$ is just the lower boundary of the set $C$ along the direction of the $(K+1)$ st coordinate in $\mathbb{R}^{K+1}$.

Before continuing to the main theorem, we state an important result known as Carathéodory's theorem [43. This theorem uses the notion of a generalized simplex of dimension d, which is just the convex hull of a set of $d+1$ affinely independent points. A triangle, or example, regardless of shape, is a generalized simplex of dimension 2. For convenience we refer to a generalized simplex as just a simplex.

Theorem 1 (Carathéodory) Let $f$ be any bounded set of points in $\mathbb{R}^{K+1}$, and let $C=\operatorname{co}[f]$ be the convex hull of $f$ (as a set). Then $\mathbf{x} \in C$ if and only if $\mathbf{x}$ can be written as a convex combination of $K+2$ (not necessarily distinct) points in $f$. Furthermore, $C$ is the union of all the simplices with dimension less than or equal to $K+1$ whose vertices belong to $f$.

From Carathéodory's theorem and the fact that the function $c$ is the boundary of the set $C$, we know that $c$ can be expressed as the union of many simplices (usually infinitely many) whose vertices belong to $f$. These simplices are necessarily of dimension at most $K$, since the dimension of $c$ is $K$. We can speak meaningfully about directional derivatives on these simplices and on $c$ because of the following beautiful fact: any convex function has well defined one-sided directional derivatives everywhere and, furthermore, is differentiable everywhere except possibly a set of measure zero [43].

Theorem 2 Let $f: \mathcal{D} \mapsto[0,1]$ be a monotone, and let $c: \mathcal{D}^{\prime} \mapsto[0,1]$ be the convex roof of the function $f$. Then $c$ is also a monotone.

Proof: The domain $\mathcal{D}^{\prime}$ of $c$ in general contains the domain $\mathcal{D}$ of $f$, but it will remain bounded and in the positive orthant of $\mathbb{R}^{K}$ and is furthermore always convex even if $\mathcal{D}$ is not. Clearly $0 \in \mathcal{D}^{\prime}$, since $0 \in \mathcal{D}$. The fact that $c(0)=0$ can be seen by the fact that $f(0)=0$ is the global minimum for $f$, and the convex hull of a function will always contain the function's global minimum. This shows that $c$ satisfies the first two criteria of a monotone.

Now we prove the final criterion, the monotonicity of $c$. Consider the set of all possible simplices with dimension less than or equal to $K$ with vertices lying in $f$. From Carathéodory's theorem, $c$ is a union of some subset of these simplices. However, every simplex in this set has the property 
of monotonicity over its domain of definition. This follows from the "multidirectional" version of the mean value theorem [44, 44, for which we now sketch the proof. Suppose we choose a simplex $s \in c$. Along a given direction $\mathbf{p}$, the smallest value of the directional derivative of $f$ lying above $s$ is a lower bound on the directional derivative of $s$. In particular, if $\mathbf{p} \geq 0$, then from the assumption of monotonicity of $f$, we know that $\nabla_{\mathbf{p}} f \geq 0$ everywhere, and hence $\nabla_{\mathbf{p}} s \geq 0$. This implies that each constituent simplex in $c$ is indeed monotonic. To show that $c$ is a monotone, we use the convexity of $c$ to see that the directional derivative in some direction $\mathbf{p} \geq 0$ across two neighboring simplices $s_{1}$ and $s_{2}$ cannot decrease.

\section{APPENDIX C: $\hat{n}_{\Phi}$ FOR $D \times N$ PURE STATES}

Our objective in this Appendix is to characterize the eigenvalues of the operator $(I \otimes \Phi) \rho_{A B}=\mathcal{O}$ in Eq. (3.11) for the special case in which the Schmidt basis for subsystem $B$ of the pure state $\rho_{A B}$ is the same as the angular-momentum basis. Recall that in Eq. (3.11), the Schmidt coefficients $\mu_{i}$ are ordered from largest to smallest. The operator $\mathcal{O}$ is a $D N \times D N$ operator, although it clearly has rank at most $D^{2}$, so we can regard it as a $D^{2} \times D^{2}$ operator, having $D^{2}$ eigenvalues. Although $\mathcal{O}$ can be written in matrix form in the Schmidt basis, we refrain from doing so here, as the expression is unwieldy and not very illuminating. We can, however, by permuting the rows and columns of $\mathcal{O}$, write it as

$$
\mathcal{O}=\mathbf{0} \oplus \mathbf{T} \oplus \mathbf{R}
$$

where $\mathbf{0}$ is a matrix of zeros, of size $D \times D$.

To describe $\mathbf{T}$ and $\mathbf{R}$, we first make some definitions. An index is an integer between 1 and $D$. An ordered pair of indices $(j, k)$ is said to be inadmissible if $k=D-j+1$ or $k=j$. All other indices are said to be admissible. A set of indices is called admissible if the elements are pairwise admissible. A product of $n$ distinct Schmidt coefficients $\mu_{j_{1}} \mu_{j_{2}} \cdots \mu_{j_{n}}$ is said to be $n$-admissible if all of the indices are pairwise admissible, and if, in addition, $j_{1}<j_{2}<\ldots<j_{n}$. Finally, define $\mathcal{S}_{n}$ as the sum over all $n$-admissible products. Then

$$
\mathbf{T}=\underset{\substack{(p, q) \\ \text { admissible }}}{ } W_{(p, q)},
$$

where each $W_{(p, q)}$ is a $2 \times 2$ matrix of the form

$$
W_{(p, q)}=\left(\begin{array}{cc}
\mu_{p} & (-1)^{p+q-1} \sqrt{\mu_{p} \mu_{q}} \\
(-1)^{p+q-1} \sqrt{\mu_{p} \mu_{q}} & \mu_{q}
\end{array}\right) .
$$

For each index, there are $D-2$ other indices with which it can form an admissible pair. Hence, $D$ indices form exactly $D(D-2) / 2$ distinct admissible pairs, and that is the number of possible $W_{(p, q)}$ 's of the given form. $W_{(p, q)}$ has eigenvalues 0 and $\mu_{p}+\mu_{q}$. Thus, $\mathbf{T}$ has $D(D-2) / 2$ zero eigenvalues and an equal number of eigenvalues $\mu_{p}+\mu_{q}$, where $(p, q)$ is an admissible pair.

The matrix $\mathbf{R}$ has elements

$$
\mathbf{R}_{j k}=-\sqrt{\mu_{j} \mu_{k}}\left(1-\delta_{j, k}\right)\left(1-\delta_{j, D-k+1}\right),
$$


where $j, k=1, \cdots, D$. It is thus a $D \times D$ matrix. The characteristic polynomial of this matrix can be written as

$$
g(z)=z^{D / 2}\left(z^{D / 2}+\sum_{t=0}^{D / 2-2} t(-1)^{t} \mathcal{S}_{t+1} z^{D / 2-t-1}-(D / 2-1)(-1)^{D / 2} \mathcal{I}\right)
$$

where

$$
\mathcal{I}=\prod_{j=1}^{D / 2}\left(\mu_{j}+\mu_{D-j+1}\right)
$$

It is evident that the matrix $\mathbf{R}$ has $D / 2$ zero eigenvalues. The remaining eigenvalues are the zeroes of the function

$$
r_{D}(z)=z^{D / 2}+\sum_{t=0}^{D / 2-2} t(-1)^{t} \mathcal{S}_{t+1} z^{D / 2-t-1}-(D / 2-1)(-1)^{D / 2} \mathcal{I} .
$$

The Descartes rule of signs tells us that the above equation has no more than one negative root. In fact, if all the Schmidt coefficients are nonzero, there is exactly one negative eigenvalue, the negative root of $r_{D}(z)$. Otherwise, all the eigenvalues are nonnegative, and the pure state under consideration could be separable.

Putting all this together, we conclude that the spectrum of $\mathcal{O}$ has

1. $D+D(D-2) / 2+D / 2=D(D+1) / 2$ zero eigenvalues,

2. $D(D-2) / 2$ positive eigenvalues of the form $\mu_{p}+\mu_{q}$, where $(p, q)$ is an admissible pair, and $D / 2-1$ positive eigenvalues, which are the positive roots of $r_{D}(z)=0$,

3. One negative eigenvalue, the negative root of $r_{D}(z)=0$.

As an example, we present the case of $D=4$. Then Eq. (C7) becomes $r_{4}(z) \equiv z^{2}-\left(\mu_{1}+\right.$ $\left.\mu_{4}\right)\left(\mu_{2}+\mu_{3}\right)$, which has zeroes $\pm \sqrt{\left(\mu_{1}+\mu_{4}\right)\left(\mu_{2}+\mu_{3}\right)}$.

For $D=6$, the function $\mathrm{C} 7 \mathrm{i}$

$$
\begin{aligned}
r_{6}(z) \equiv z^{3} & -z\left(\mu_{1} \mu_{2}+\mu_{1} \mu_{3}+\mu_{2} \mu_{3}+\mu_{1} \mu_{4}+\mu_{2} \mu_{4}+\mu_{1} \mu_{5}+\mu_{3} \mu_{5}+\mu_{4} \mu_{5}\right. \\
& \left.+\mu_{2} \mu_{6}+\mu_{3} \mu_{6}+\mu_{4} \mu_{6}+\mu_{5} \mu_{6}\right)+2\left(\mu_{1}+\mu_{6}\right)\left(\mu_{2}+\mu_{5}\right)\left(\mu_{3}+\mu_{4}\right) .
\end{aligned}
$$

\section{APPENDIX D: SOLUTIONS OF THE CONSTRAINT EQUATIONS}

The three constraint equations,

$$
\begin{aligned}
\frac{1}{2}\left[\left(\sqrt{\mu_{1}}+\sqrt{\mu_{2}}+\sqrt{\mu_{3}}+\sqrt{\mu_{4}}\right)^{2}-1\right] & =n_{T}, \\
3 \sqrt{\left(\mu_{1}+\mu_{4}\right)\left(\mu_{2}+\mu_{3}\right)} & =\hat{n}_{\Phi}, \\
\mu_{1}+\mu_{2}+\mu_{3}+\mu_{4} & =1,
\end{aligned}
$$


can be solved to express $\mu_{1}, \mu_{2}$, and $\mu_{3}$ in terms of $n_{T}, \hat{n}_{\Phi}$, and $\mu_{4}$. There are four sets of solutions, of which only two are distinct because the other two can be obtained by exchanging $\mu_{2}$ and $\mu_{3}$. The constraint equations are invariant under this exchange. The two distinct solutions are the following:

$$
\begin{aligned}
& \mu_{1}^{(1)}=\frac{1}{2}\left(1+\sqrt{1-\frac{4}{9} \hat{n}_{\Phi}^{2}}-2 \mu_{4}\right) \\
& \mu_{2}^{(1)}=\frac{1}{4}\left(1-\sqrt{1-\frac{4}{9} \hat{n}_{\Phi}^{2}}+2 \sqrt{\mathcal{G}_{0}-\mathcal{G}_{1}\left(\mu_{1}^{(1)}\right)}\right) \\
& \mu_{3}^{(1)}=\frac{1}{4}\left(1-\sqrt{1-\frac{4}{9} \hat{n}_{\Phi}^{2}}-2 \sqrt{\mathcal{G}_{0}-\mathcal{G}_{1}\left(\mu_{1}^{(1)}\right)}\right)
\end{aligned}
$$

and

$$
\begin{aligned}
& \mu_{1}^{(2)}=\frac{1}{2}\left(1-\sqrt{1-\frac{4}{9} \hat{n}_{\Phi}^{2}}-2 \mu_{4}\right), \\
& \mu_{2}^{(2)}=\frac{1}{4}\left(1+\sqrt{1-\frac{4}{9} \hat{n}_{\Phi}^{2}}+2 \sqrt{\mathcal{G}_{0}-\mathcal{G}_{1}\left(\mu_{1}^{(2)}\right)}\right), \\
& \mu_{3}^{(2)}=\frac{1}{4}\left(1+\sqrt{1-\frac{4}{9} \hat{n}_{\Phi}^{2}}-2 \sqrt{\mathcal{G}_{0}-\mathcal{G}_{1}\left(\mu_{1}^{(2)}\right)}\right) .
\end{aligned}
$$

Here $\mathcal{G}_{0}$ and $\mathcal{G}_{1}$ are given by

$$
\begin{gathered}
\mathcal{G}_{0}=1+8\left(n_{T}+\mu_{4}\right) \sqrt{\mu_{4}\left(2 n_{T}+1\right)}-4 n_{T}\left(n_{T}+4 \mu_{4}\right)-3 \mu_{4}\left(\mu_{4}+2\right), \\
\mathcal{G}_{1}\left(\mu_{1}\right)=\frac{\mu_{1}^{2}}{12}+\left(\frac{2 \mu_{1}}{3}\right)^{3 / 2}\left(\sqrt{2 n_{T}+1}-\sqrt{\mu_{4}}\right)+\frac{\mu_{1}}{3}\left(3+8 n_{T}-8 \sqrt{\mu_{4}\left(2 n_{T}+1\right)}+5 \mu_{4}\right) \\
+\frac{4 \sqrt{6 \mu_{1}}}{3}\left[\sqrt{\mu_{4}}\left(1-2 \sqrt{\mu_{4}\left(2 n_{T}+1\right)}+\mu_{4}\right)-n_{T}\left(\sqrt{2 n_{T}+1}-3 \sqrt{\mu_{4}}\right)\right] .
\end{gathered}
$$

\section{ACKNOWLEDGEMENTS}

The authors thank M. Horodecki, A. Denney, S. Merkel, and A. Silberfarb for useful discussions. This work was supported in part by Office of Naval Research Contract No. N00014-03-1-0426.

[1] E. Schrödinger, Proc. Camb. Phil. Soc 31, 555 (1935).

[2] D. Bruß, J. Math. Phys. 43, 4237 (2002).

[3] M. A. Nielsen and I. L. Chuang, Quantum Computation and Quantum Information (Cambridge University Press, Cambridge, 2000).

[4] A. Einstein, B. Podolsky, and N. Rosen, Phys. Rev. 47, 777 (1935).

[5] J. S. Bell, Physics 1, 195 (1964).

[6] S. Ghosh, T. F. Rosenbaum, G. Aeppli, and S. N. Coppersmith, Nature 425, 48 (2003). 
[7] T. Osborne and M. A. Neilsen, Quant. Info. Process. 1, 45 (2002).

[8] A. Osterloh, L. Amico, G. Falci, and R. Fazio, Nature 416, 608 (2002).

[9] G. Vidal, J. I. Latorre, E. Rico, and A. Kitaev, Phys. Rev. Lett. 90, 227902 (2003).

[10] L. Gurvits, in Proceedings of the thirty-fifth ACM symposium on Theory of computing (ACM press, New York, 2003), p. 10.

[11] H.-P. Breuer, Phys. Rev. Lett. 97, 080501 (pages 4) (2006).

[12] K. Chen, S. Albeverio, and S.-M. Fei, Phys. Rev. Lett. 95, 40504 (2005).

[13] C. H. Bennett, H. J. Bernstein, S. Popescu, and B. Schumacher, Phys. Rev. A 53, 2046 (1996).

[14] P. Hayden, M. Horodecki, and B. M. Terhal, J. Phys. A: Math. Gen. 34, 6891 (2001).

[15] W. K. Wootters, Phys. Rev. Lett. 80, 2245 (1998).

[16] P. Rungta, V. Buzek, C. M. Caves, M. Hillery, and G. J. Milburn, Phys. Rev. A 64, 42315 (2001).

[17] P. Rungta and C. M. Caves, Phys. Rev. A 67, 12307 (2003).

[18] G. Vidal, J. Mod. Opt. 47, 355 (2000).

[19] W. F. Stinespring, Proc. Am. Math. Soc. 6, 211 (1955).

[20] E. Størmer, Acta Math. 110, 233 (1963).

[21] M.-D. Choi, Can. J. Math. 24, 520 (1972).

[22] M.-D. Choi, Illinois J. Math. 18, 565 (1974).

[23] M.-D. Choi, Linear Algebra Appl. 10, 285 (1975).

[24] M. Horodecki, P. Horodecki, and R. Horodecki, Phys. Lett. A 223, 1 (1996).

[25] B. M. Terhal, Linear Algebra Appl. 323, 61 (2001).

[26] O. Rudolph, J. Math. Phys. 33, 3951 (2000).

[27] O. Rudolph, arXiv:quant-ph/0202121 (2002).

[28] K. Chen and L. A. Wu, Quant. Inf. Comput. 3, 193 (2003).

[29] M. Plenio and S. Virmani, Quant. Inf. Comput. 7, 1 (2007).

[30] G. Vidal and R. F. Werner, Phys. Rev. A 65, 032314 (2002).

[31] K. Zyczkowski, P. Horodecki, A. Sanpera, and M. Lewenstein, Physical Review A 58, 883 (1998).

[32] A. Peres, Phys. Rev. Lett. 77, 1413 (1996).

[33] K. Chen, S. Albeverio, and S.-M. Fei, Phys. Rev. Lett. 95, 210501 (2005).

[34] H.-P. Breuer, J. Phys. A: Math. Gen. 39, 11847 (2006).

[35] O. Gühne, M. Reimpell, and R. F. Werner, arXiv:quant-ph/0607163 (2006).

[36] J. Eisert, F. G. S. L. Brandão, and K. M. R. Audenaert, arXiv:quant-ph/0607167 (2006).

[37] N. J. Cerf, C. Adami, and R. M. Gingrich, Phys. Rev. A 60, 898 (1999).

[38] M. Horodecki and P. Horodecki, Phys. Rev. A 59, 4206 (1999).

[39] H.-P. Breuer, Phys. Rev. A 71, 062330 (2005).

[40] M. Lewenstein, B. Kraus, J. I. Cirac, and P. Horodecki, Phys. Rev. A 62, 52310 (2000).

[41] B. M. Terhal and K. G. H. Vollbrecht, Phys. Rev. Lett. 85, 2625 (2000).

[42] S.-M. Fei and X. Li-Jost, Phys. Rev. A 73, 24302 (2006).

[43] T. R. Rockafellar, Convex Analysis, Princeton landmarks in mathematics (Princeton University Press, Princeton, New Jersey, 1997).

[44] F. H. Clarke and Y. S. Ledyaev, Trans. Am. Math. Soc. 344, 307 (1994).

[45] F. H. Clarke and Y. S. Ledyaev, Proc. Am. Math. Soc. 122, 1075 (1994). 